\title{
Fuzzy Soft Expert Set and Its Application
}

\author{
Shawkat Alkhazaleh1, Abdul Razak Salleh2* \\ ${ }^{1}$ Department of Mathematics, Faculty of Science and Art, Shaqra University, Shaqraa, KSA \\ ${ }^{2}$ School of Mathematical Sciences, Faculty of Science and Technology, Universiti Kebangsaan Malaysia, Bangi, \\ Malaysia \\ Email: shmk79@gmail.com, ${ }^{*}$ aras@ukm.my
}

Received 27 February 2014; revised 27 March 2014; accepted 3 April 2014

Copyright (C) 2014 by authors and Scientific Research Publishing Inc.

This work is licensed under the Creative Commons Attribution International License (CC BY).

http://creativecommons.org/licenses/by/4.0/

(c) (i) Open Access

\begin{abstract}
In 1999, Molodtsov introduced the concept of soft set theory as a general mathematical tool for dealing with uncertainty. Alkhazaleh and Salleh (2011) define the concept of soft expert sets where the user can know the opinion of all experts in one model and give an application of this concept in decision making problem. So in this paper, we generalize the concept of a soft expert set to fuzzy soft expert set, which will be more effective and useful. We also define its basic operations, namely complement, union, intersection, AND and OR. We give an application of this concept in decision making problem. Finally, we study a mapping on fuzzy soft expert classes and its properties.
\end{abstract}

\section{Keywords}

\section{Soft Set, Soft Expert Set, Fuzzy Soft Expert Set}

\section{Introduction}

Many scientists wish to find appropriate solutions to some mathematical problems that cannot be solved by traditional methods. These problems lie in the fact that traditional methods cannot solve the problems of uncertainty in economy, engineering, medicine and the problems of decision-making and others. One of these solutions is fuzzy sets- the title of Zadeh's first article about his new mathematical theory, which was published in a scientific journal in 1965. Since Zadeh published his new classic paper almost fifty years ago, fuzzy set theory has received more and more attention from researchers in a wide range of scientific areas, especially in the past few years. The difference between a binary set and a fuzzy set is that in a "normal" set every element is either a member or a non-member of the set. Here, we see that it either has to be $A$ or not $A$. In a fuzzy set, an element can be a member of a set to some degree and at the same time a non-member to some degree of the same set. In

${ }^{*}$ Corresponding author. 
classical set theory, the membership of elements in a set is assessed in binary terms according to a bivalent condition; an element either belongs or does not belong to the set. By contrast, fuzzy set theory permits the gradual assessment of the membership of elements in a set; this is described with the aid of a membership function valued in the closed unit interval [0,1]. Fuzzy sets generalise classical sets, since the indicator functions of classical sets are special cases of the membership functions of fuzzy sets, if the latter only take values 0 or 1 . Therefore, a fuzzy set $A$ in a universe of discourse $X$ is a function $A: X \rightarrow[0,1]$, usually this function is referred to as the membership function and denoted by $\mu_{A}(x)$. Some mathematicians use the notation $A(x)$ to denote the membership function instead of $\mu_{A}(x)$. A fuzzy set $A$ is written symbolically in various

$$
A=\left\{\left\langle x, \mu_{A}(x)\right\rangle: x \in X\right\} \text {. }
$$

Molodtsov [1] initiated the concept of soft set theory as a mathematical tool for dealing with uncertainties. After Molodtsov's work, some operations and application of soft sets were studied by Chen et al. [2], Maji et al. [3] and Maji et al. [4]. Also Maji et al. [5] have introduced the concept of fuzzy soft set, a more general concept, which is a combination of fuzzy set and soft set and studied its properties and also Roy and Maji [6] used this theory to solve some decision making problems. Alkhazaleh et al. [7] introduced the concept of soft multisets as a generalization of soft set. They also defined the concepts of fuzzy parameterized interval-valued fuzzy soft set [8] and possibility fuzzy soft set [9] and gave their applications in decision making and medical diagnosis. Alkhazaleh and Salleh [10] introduced the concept of a soft expert set, where the user can know the opinion of all experts in one model without any operations. Even after any operation the user can know the opinion of all experts. So in this paper, we introduce the concept of a fuzzy soft expert set, which will be more effective and useful and which is a combination of fuzzy set and soft expert set. We also define its basic operations, namely complement, union, intersection, AND and OR and study their properties. We give an application of this concept in decision making problem. Finally, we study a mapping on fuzzy soft expert classes and its properties.

\section{Preliminaries}

In this section, we recall some basic notions related to this work. Molodtsov defined soft set in the following way. Let $U$ be a universe and $E$ be a set of parameters. Let $P(U)$ denote the power set of $U$ and $A \subseteq E$.

Definition $1[1]$ A pair $(F, A)$ is called a soft set over $U$ where $F$ is a mapping $F: A \rightarrow P(U)$.

In other words, a soft set over $U$ is a parameterized family of subsets of the universe $U$ For $\varepsilon \in A, F(\varepsilon)$ may be considered as the set of $\varepsilon$-approximate elements of the soft set $(F, A)$.

Definition 2 [5] Let $U$ be an initial universal set and let $E$ be a set of parameters. Let $I^{U}$ denote the power set of all fuzzy subsets of $U$. Let $A \subseteq E$. A pair $(F, E)$ is called a fuzzy soft set over $U$ where $F$ is a mapping given by

$$
F: A \rightarrow I^{U} \text {. }
$$

Let $U$ be a universe, $E$ a set of parameters, $X$ a set of experts (agents), and $O$ a set of opinions. Let $Z=E \times X \times$ $O$ and $A \subseteq Z$.

Definition $3[10]$ A pair $(F, A)$ is called a soft expert set over $U$, where $F$ is a mapping given by

$$
F: A \rightarrow P(U)
$$

where $P(U)$ denotes the power set of $U$.

Definition 4 [10] For two soft expert sets $(F, A)$ and $(G, B)$ over $U,(F, A)$ is called a soft expert subset of $(G, B)$ if

1. $A \subseteq B$,

2. $\forall \varepsilon \in A, F(\varepsilon) \subseteq G(\varepsilon)$.

This relationship is denoted by $(F, A) \tilde{\subseteq}(G, B)$. In this case $(G, B)$ is called a soft expert superset of $(F, A)$.

Definition 5 [10] Two soft expert sets $(F, A)$ and $(G, B)$ over $U$ are said to be equal if $(F, A)$ is a soft expert subset of $(G, B)$ and $(G, B)$ is a soft expert subset of $(F, A)$

Definition 6 [10] Let $E$ be a set of parameters and $X$ a set of experts. The NOT set of $Z=E \times X \times O$, denoted by $\rceil Z$, is defined by $\left.\rceil Z=\{\rceil e_{i} \times x_{j} \times o_{k}, \forall i, j, k\right\}$.

Definition 7 [10] An agree-soft expert set $(F, A)_{1}$ over $U$ is a soft expert subset of $(F, A)$ defined as follows:

$$
(F, A)_{1}=\left\{F_{1}(\alpha): \alpha \in E \times X \times\{1\}\right\} .
$$


Definition 8 [10] A disagree-soft expert set $(F, A)_{0}$ over $U$ is a soft expert subset of $(F, A)$ defined as follows:

$$
(F, A)_{0}=\left\{F_{0}(\alpha): \alpha \in E \times X \times\{0\}\right\} .
$$

Definition 9 [10] The complement of a soft expert set $(F, A)$ is denoted by $(F, A)^{c}$ and is defined by $\left.(F, A)^{c}=\left(F^{c},\right\rceil A\right)$ where $\left.F^{c}:\right\rceil A \rightarrow P(U)$ is a mapping given by

$$
\left.\left.F^{c}(\alpha)=U-F(\rceil \alpha\right), \forall \alpha \in\right\rceil A \text {. }
$$

Definition 10 [10] The union of two soft expert sets $(F, A)$ and $(G, B)$ over $U$, denoted by $(F, A) \tilde{\cup}(G, B)$, is the soft expert set $(H, C)$ where $C=A \cup B$, and $\forall \varepsilon \in C$,

$$
H(\varepsilon)= \begin{cases}F(\varepsilon), & \text { if } \varepsilon \in A-B \\ G(\varepsilon), & \text { if } \varepsilon \in B-A \\ F(\varepsilon) \cup G(\varepsilon), & \text { if } \varepsilon \in A \cap B .\end{cases}
$$

Definition 11 [10] The intersection of two soft expert sets $(F, A)$ and $(G, B)$ over $U$, denoted by $(F, A) \tilde{\cap}(G, B)$, is the soft expert set $(H, C)$ where $C=A \cup B$, and $\forall \varepsilon \in C$,

$$
H(\varepsilon)= \begin{cases}F(\varepsilon), & \text { if } \varepsilon \in A-B \\ G(\varepsilon), & \text { if } \varepsilon \in B-A \\ F(\varepsilon) \cap G(\varepsilon), & \text { if } \varepsilon \in A \cap B .\end{cases}
$$

Definition $12[10]$ If $(F, A)$ and $(G, B)$ are two soft expert sets over $U$ then " $(F, A)$ AND $(G, B)$ " denoted by $(F, A) \wedge(G, B)$, is defined by

$$
(F, A) \wedge(G, B)=(H, A \times B)
$$

where $H(\alpha, \beta)=F(\alpha) \cap G(\beta), \forall(\alpha, \beta) \in A \times B$.

Definition 13 [10] If $(F, A)$ and $(G, B)$ are two soft expert sets over $U$ then " $(F, A)$ OR $(G, B)$ " denoted by $(F, A) \vee(G, B)$, is defined by

$$
(F, A) \vee(G, B)=(H, A \times B)
$$

where $H(\alpha, \beta)=F(\alpha) \cup G(\beta), \forall(\alpha, \beta) \in A \times B$.

\section{Fuzzy Soft Expert Set}

In this section, we introduce the definition of a fuzzy soft expert set and give basic properties of this concept.

Let $U$ be a universe, $E$ a set of parameters, $X$ a set of experts (agents), and $O=\{1=$ agree, $0=$ disagree $\}$ a set of opinions. Let $Z=E \times X \times O$ and $A \subseteq Z$.

Definition 14 A pair $(F, A)$ is called a fuzzy soft expert set over $U$, where $F$ is a mapping given by

$$
F: A \rightarrow I^{U}
$$

where $I^{U}$ denotes the set of all fuzzy subsets of $U$.

Example 1 Suppose that a company produces new types of products and wants to take the opinion of some experts about these products. Let $U=\left\{u_{1}, u_{2}, u_{3}, u_{4}\right\}$ be a set of products, $E=\left\{e_{1}, e_{2}, e_{3}\right\}$ is a set of decision parameters where $e_{i}(i=1,2,3)$ denotes the parameters "easy to use", "quality" and "cheap". Let $X=\{p, q, r\}$ be a set of experts. Suppose that

$$
\begin{aligned}
& F\left(e_{1}, p, 1\right)=\left\{\frac{u_{1}}{0.3}, \frac{u_{2}}{0.5}, \frac{u_{3}}{0.7}, \frac{u_{4}}{0.1}\right\}, \quad F\left(e_{1}, q, 1\right)=\left\{\frac{u_{1}}{0.5}, \frac{u_{2}}{0.2}, \frac{u_{3}}{0.3}, \frac{1}{u_{4}}\right\}, \\
& F\left(e_{1}, r, 1\right)=\left\{\frac{u_{1}}{0.4}, \frac{u_{2}}{0.8}, \frac{u_{3}}{0.3}, \frac{u_{4}}{0.4}\right\}, \quad F\left(e_{2}, p, 1\right)=\left\{\frac{u_{1}}{0.3}, \frac{u_{2}}{0.2}, \frac{u_{3}}{0.5}, \frac{u_{4}}{0.6}\right\}, \\
& F\left(e_{2}, q, 1\right)=\left\{\frac{u_{1}}{0.6}, \frac{u_{2}}{0.4}, \frac{u_{3}}{0.3}, \frac{u_{4}}{0.7}\right\}, \quad F\left(e_{2}, r, 1\right)=\left\{\frac{u_{1}}{0.1}, \frac{u_{2}}{0.3}, \frac{u_{3}}{0.7}, \frac{u_{4}}{0.4}\right\},
\end{aligned}
$$




$$
\begin{aligned}
& F\left(e_{3}, p, 1\right)=\left\{\frac{u_{1}}{0.6}, \frac{u_{2}}{0.2}, \frac{u_{3}}{0.4}, \frac{u_{4}}{0.3}\right\}, \quad F\left(e_{3}, q, 1\right)=\left\{\frac{u_{1}}{0.5}, \frac{u_{2}}{0.3}, \frac{u_{3}}{0.5}, \frac{u_{4}}{0.7}\right\}, \\
& F\left(e_{3}, r, 1\right)=\left\{\frac{u_{1}}{0.3}, \frac{u_{2}}{0.4}, \frac{u_{3}}{0.3}, \frac{u_{4}}{0.4}\right\}, \quad F\left(e_{1}, p, 0\right)=\left\{\frac{u_{1}}{0.6}, \frac{u_{2}}{0.4}, \frac{u_{3}}{0.4}, \frac{u_{4}}{0.6}\right\}, \\
& F\left(e_{1}, q, 0\right)=\left\{\frac{u_{1}}{0.3}, \frac{u_{2}}{0.6}, \frac{u_{3}}{0.4}, \frac{0}{u_{4}}\right\}, \quad F\left(e_{1}, r, 0\right)=\left\{\frac{u_{1}}{0.5}, \frac{u_{2}}{0.1}, \frac{u_{3}}{0.7}, \frac{u_{4}}{0.5}\right\}, \\
& F\left(e_{2}, p, 0\right)=\left\{\frac{u_{1}}{0.5}, \frac{u_{2}}{0.6}, \frac{u_{3}}{0.4}, \frac{u_{4}}{0.2}\right\}, \quad F\left(e_{2}, q, 0\right)=\left\{\frac{u_{1}}{0.2}, \frac{u_{2}}{0.4}, \frac{u_{3}}{0.5}, \frac{u_{4}}{0.1}\right\}, \\
& F\left(e_{2}, r, 0\right)=\left\{\frac{u_{1}}{0.7}, \frac{u_{2}}{0.5}, \frac{u_{3}}{0.2}, \frac{u_{4}}{0.5}\right\}, \quad F\left(e_{3}, p, 0\right)=\left\{\frac{u_{1}}{0.3}, \frac{u_{2}}{0.5}, \frac{u_{3}}{0.5}, \frac{u_{4}}{0.6}\right\}, \\
& F\left(e_{3}, q, 0\right)=\left\{\frac{u_{1}}{0.3}, \frac{u_{2}}{0.5}, \frac{u_{3}}{0.4}, \frac{u_{4}}{0.2}\right\}, \quad F\left(e_{3}, r, 0\right)=\left\{\frac{u_{1}}{0.5}, \frac{u_{2}}{0.4}, \frac{u_{3}}{0.6}, \frac{u_{4}}{0.5}\right\} .
\end{aligned}
$$

Then we can view the fuzzy soft expert set $(F, Z)$ as consisting of the following collection of approximations:

$$
\begin{aligned}
(F, Z)=\{ & \left(\left(e_{1}, p, 1\right),\left\{\frac{u_{1}}{0.3}, \frac{u_{2}}{0.5}, \frac{u_{3}}{0.7}, \frac{u_{4}}{0.1}\right\}\right),\left(\left(e_{1}, q, 1\right),\left\{\frac{u_{1}}{0.5}, \frac{u_{2}}{0.2}, \frac{u_{3}}{0.3}, \frac{1}{u_{4}}\right\}\right), \\
& \left(\left(e_{1}, r, 1\right),\left\{\frac{u_{1}}{0.4}, \frac{u_{2}}{0.8}, \frac{u_{3}}{0.3}, \frac{u_{4}}{0.4}\right\}\right),\left(\left(e_{2}, p, 1\right),\left\{\frac{u_{1}}{0.3}, \frac{u_{2}}{0.2}, \frac{u_{3}}{0.5}, \frac{u_{4}}{0.6}\right\}\right), \\
& \left(\left(e_{2}, q, 1\right),\left\{\frac{u_{1}}{0.6}, \frac{u_{2}}{0.4}, \frac{u_{3}}{0.3}, \frac{u_{4}}{0.7}\right\}\right),\left(\left(e_{2}, r, 1\right),\left\{\frac{u_{1}}{0.1}, \frac{u_{2}}{0.3}, \frac{u_{3}}{0.7}, \frac{u_{4}}{0.4}\right\}\right), \\
& \left(\left(e_{3}, p, 1\right),\left\{\frac{u_{1}}{0.6}, \frac{u_{2}}{0.2}, \frac{u_{3}}{0.4}, \frac{u_{4}}{0.3}\right\}\right),\left(\left(e_{3}, q, 1\right),\left\{\frac{u_{1}}{0.5}, \frac{u_{2}}{0.3}, \frac{u_{3}}{0.5}, \frac{u_{4}}{0.7}\right\}\right), \\
& \left(\left(e_{3}, r, 1\right),\left\{\frac{u_{1}}{0.3}, \frac{u_{2}}{0.4}, \frac{u_{3}}{0.3}, \frac{u_{4}}{0.4}\right\}\right),\left(\left(e_{1}, p, 0\right),\left\{\frac{u_{1}}{0.6}, \frac{u_{2}}{0.4}, \frac{u_{3}}{0.4}, \frac{u_{4}}{0.6}\right\}\right), \\
& \left(\left(e_{1}, q, 0\right),\left\{\frac{u_{1}}{0.3}, \frac{u_{2}}{0.6}, \frac{u_{3}}{0.4}, \frac{0}{u_{4}}\right\}\right),\left(\left(e_{1}, r, 0\right),\left\{\frac{u_{1}}{0.5}, \frac{u_{2}}{0.1}, \frac{u_{3}}{0.7}, \frac{u_{4}}{0.5}\right\}\right), \\
& \left(\left(e_{2}, p, 0\right),\left\{\frac{u_{1}}{0.5}, \frac{u_{2}}{0.6}, \frac{u_{3}}{0.4}, \frac{u_{4}}{0.2}\right\}\right),\left(\left(e_{2}, q, 0\right),\left\{\frac{u_{1}}{0.2}, \frac{u_{2}}{0.4}, \frac{u_{3}}{0.5}, \frac{u_{4}}{0.1}\right\}\right), \\
& \left(\left(e_{2}, r, 0\right),\left\{\frac{u_{1}}{0.7}, \frac{u_{2}}{0.5}, \frac{u_{3}}{0.2}, \frac{u_{4}}{0.5}\right\}\right),\left(\left(e_{3}, p, 0\right),\left\{\frac{u_{1}}{0.3}, \frac{u_{2}}{0.5}, \frac{u_{3}}{0.5}, \frac{u_{4}}{0.6}\right\}\right), \\
& \left.\left(\left(e_{3}, q, 0\right),\left\{\frac{u_{1}}{0.3}, \frac{u_{2}}{0.5}, \frac{u_{3}}{0.4}, \frac{u_{4}}{0.2}\right\}\right),\left(\left(e_{3}, r, 0\right),\left\{\frac{u_{1}}{0.5}, \frac{u_{2}}{0.4}, \frac{u_{3}}{0.6}, \frac{u_{4}}{0.5}\right\}\right)\right\} .
\end{aligned}
$$

Definition 15 For two fuzzy soft expert sets $(F, A)$ and $(G, B)$ over $U,(F, A)$ is called a fuzzy soft expert subset of $(G, B)$ if

1. $B \subseteq A$,

2. $\forall \varepsilon \in A, F(\varepsilon)$ is fuzzy subset of $G(\varepsilon)$.

This relationship is denoted by $(F, A) \tilde{\subseteq}(G, B)$. In this case $(G, B)$ is called a fuzzy soft expert superset of $(F, A)$.

Definition 16 Two fuzzy soft expert sets $(F, A)$ and $(G, B)$ over $U$ are said to be equal if $(F, A)$ is a fuzzy soft expert subset of $(G, B)$ and $(G, B)$ is a fuzzy soft expert subset of $(F, A)$.

Example 2 Consider Example 1. Suppose that the company takes the opinion of the experts once again after a month of using the products. Let 


$$
A=\left\{\left(e_{1}, p, 1\right),\left(e_{2}, p, 0\right),\left(e_{3}, p, 1\right),\left(e_{1}, q, 1\right),\left(e_{2}, q, 1\right),\left(e_{1}, r, 0\right),\left(e_{2}, r, 1\right),\left(e_{3}, r, 1\right)\right\}
$$

and

$$
B=\left\{\left(e_{1}, p, 1\right),\left(e_{2}, p, 0\right),\left(e_{1}, q, 1\right),\left(e_{2}, q, 1\right),\left(e_{1}, r, 0\right),\left(e_{2}, r, 1\right)\right\}
$$

Clearly $B \subseteq A$. Let $(F, A)$ and $(G, B)$ be defined as follows:

$$
\begin{aligned}
&(F, A)=\{\left(\left(e_{1}, p, 1\right),\left\{\frac{u_{1}}{0.3}, \frac{u_{2}}{0.5}, \frac{u_{3}}{0.4}, \frac{u_{4}}{0.2}\right\}\right),\left(\left(e_{1}, q, 1\right),\left\{\frac{u_{1}}{0.5}, \frac{u_{2}}{0.4}, \frac{u_{3}}{0.3}, \frac{u_{4}}{0.6}\right\}\right), \\
&\left(\left(e_{2}, q, 1\right),\left\{\frac{u_{1}}{0.6}, \frac{u_{2}}{0.2}, \frac{u_{3}}{0.5}, \frac{u_{4}}{0.3}\right\}\right),\left(\left(e_{2}, r, 1\right),\left\{\frac{u_{1}}{0.5}, \frac{u_{2}}{0.4}, \frac{u_{3}}{0.4}, \frac{u_{4}}{0.5}\right\}\right), \\
&\left(\left(e_{3}, p, 1\right),\left\{\frac{u_{1}}{0.7}, \frac{u_{2}}{0.4}, \frac{u_{3}}{0.3}, \frac{u_{4}}{0.4}\right\}\right),\left(\left(e_{3}, r, 1\right),\left\{\frac{u_{1}}{0.6}, \frac{u_{2}}{0.3}, \frac{u_{3}}{0.5}, \frac{u_{4}}{0.6}\right\}\right), \\
&\left.\left(\left(e_{1}, r, 0\right),\left\{\frac{u_{1}}{0.4}, \frac{u_{2}}{0.3}, \frac{u_{3}}{0.5}, \frac{u_{4}}{0.2}\right\}\right),\left(\left(e_{2}, p, 0\right),\left\{\frac{u_{1}}{0.5}, \frac{u_{2}}{0.4}, \frac{u_{3}}{0.6}, \frac{u_{4}}{0.3}\right\}\right)\right\}, \\
&(G, B)=\left\{\left(\left(e_{1}, p, 1\right),\left\{\frac{u_{1}}{0.3}, \frac{u_{2}}{0.4}, \frac{u_{3}}{0.3}, \frac{u_{4}}{0.1}\right\}\right),\left(\left(e_{1}, q, 1\right),\left\{\frac{u_{1}}{0.3}, \frac{u_{2}}{0.4}, \frac{u_{3}}{0.2}, \frac{u_{4}}{0.4}\right\}\right),\right. \\
&\left(\left(e_{2}, q, 1\right),\left\{\frac{u_{1}}{0.5}, \frac{u_{2}}{0.2}, \frac{u_{3}}{0.3}, \frac{u_{4}}{0.1}\right\}\right),\left(\left(e_{2}, r, 1\right),\left\{\frac{u_{1}}{0.3}, \frac{u_{2}}{0.2}, \frac{u_{3}}{0.2}, \frac{u_{4}}{0.3}\right\}\right), \\
&\left.\left(\left(e_{1}, r, 0\right),\left\{\frac{u_{1}}{0.3}, \frac{u_{2}}{0.3}, \frac{u_{3}}{0.3}, \frac{u_{4}}{0.1}\right\}\right),\left(\left(e_{2}, p, 0\right),\left\{\frac{u_{1}}{0.4}, \frac{u_{2}}{0.3}, \frac{u_{3}}{0.5}, \frac{u_{4}}{0.3}\right\}\right)\right\},
\end{aligned}
$$

Therefore $(G, B) \tilde{\subseteq}(F, A)$.

Definition 17 An agree-fuzzy soft expert set $(F, A)_{1}$ over $U$ is a fuzzy soft expert subset of $(F, A)$ defined as follows:

$$
(F, A)_{1}=\left\{F_{1}(\alpha): \alpha \in E \times X \times\{1\}\right\} .
$$

Definition 18 A disagree-fuzzy soft expert set $(F, A)_{0}$ over $U$ is a fuzzy soft expert subset of $(F, A)$ defined as follows:

$$
(F, A)_{0}=\left\{F_{0}(\alpha): \alpha \in E \times X \times\{0\}\right\} .
$$

Example 3 Consider Example 1. Then the agree-fuzzy soft expert set $(F, A)_{1}$ over $U$ is

$$
\begin{aligned}
&(F, Z)_{1}=\left\{\left(\left(e_{1}, p, 1\right),\left\{\frac{u_{1}}{0.3}, \frac{u_{2}}{0.5}, \frac{u_{3}}{0.7}, \frac{u_{4}}{0.1}\right\}\right),\left(\left(e_{1}, q, 1\right),\left\{\frac{u_{1}}{0.5}, \frac{u_{2}}{0.2}, \frac{u_{3}}{0.3}, \frac{1}{u_{4}}\right\}\right),\right. \\
&\left(\left(e_{1}, r, 1\right),\left\{\frac{u_{1}}{0.4}, \frac{u_{2}}{0.8}, \frac{u_{3}}{0.3}, \frac{u_{4}}{0.4}\right\}\right),\left(\left(e_{2}, p, 1\right),\left\{\frac{u_{1}}{0.3}, \frac{u_{2}}{0.2}, \frac{u_{3}}{0.5}, \frac{u_{4}}{0.6}\right\}\right), \\
&\left(\left(e_{2}, q, 1\right),\left\{\frac{u_{1}}{0.6}, \frac{u_{2}}{0.4}, \frac{u_{3}}{0.3}, \frac{u_{4}}{0.7}\right\}\right),\left(\left(e_{2}, r, 1\right),\left\{\frac{u_{1}}{0.1}, \frac{u_{2}}{0.3}, \frac{u_{3}}{0.7}, \frac{u_{4}}{0.4}\right\}\right), \\
&\left(\left(e_{3}, p, 1\right),\left\{\frac{u_{1}}{0.6}, \frac{u_{2}}{0.2}, \frac{u_{3}}{0.4}, \frac{u_{4}}{0.3}\right\}\right),\left(\left(e_{3}, q, 1\right),\left\{\frac{u_{1}}{0.5}, \frac{u_{2}}{0.3}, \frac{u_{3}}{0.5}, \frac{u_{4}}{0.7}\right\}\right), \\
&\left.\left(\left(e_{3}, r, 1\right),\left\{\frac{u_{1}}{0.3}, \frac{u_{2}}{0.4}, \frac{u_{3}}{0.3}, \frac{u_{4}}{0.4}\right\}\right)\right\},
\end{aligned}
$$

and the disagree-fuzzy soft expert set $(F, A)_{0}$ over $U$ is 


$$
\begin{aligned}
(F, Z)_{0}=\{ & \left(\left(e_{1}, p, 0\right),\left\{\frac{u_{1}}{0.6}, \frac{u_{2}}{0.4}, \frac{u_{3}}{0.4}, \frac{u_{4}}{0.6}\right\}\right),\left(\left(e_{1}, q, 0\right),\left\{\frac{u_{1}}{0.3}, \frac{u_{2}}{0.6}, \frac{u_{3}}{0.4}, \frac{0}{u_{4}}\right\}\right), \\
& \left(\left(e_{1}, r, 0\right),\left\{\frac{u_{1}}{0.5}, \frac{u_{2}}{0.1}, \frac{u_{3}}{0.7}, \frac{u_{4}}{0.5}\right\}\right),\left(\left(e_{2}, p, 0\right),\left\{\frac{u_{1}}{0.5}, \frac{u_{2}}{0.6}, \frac{u_{3}}{0.4}, \frac{u_{4}}{0.2}\right\}\right), \\
& \left(\left(e_{2}, q, 0\right),\left\{\frac{u_{1}}{0.2}, \frac{u_{2}}{0.4}, \frac{u_{3}}{0.5}, \frac{u_{4}}{0.1}\right\}\right),\left(\left(e_{2}, r, 0\right),\left\{\frac{u_{1}}{0.7}, \frac{u_{2}}{0.5}, \frac{u_{3}}{0.2}, \frac{u_{4}}{0.5}\right\}\right), \\
& \left(\left(e_{3}, p, 0\right),\left\{\frac{u_{1}}{0.3}, \frac{u_{2}}{0.5}, \frac{u_{3}}{0.5}, \frac{u_{4}}{0.6}\right\}\right),\left(\left(e_{3}, q, 0\right),\left\{\frac{u_{1}}{0.3}, \frac{u_{2}}{0.5}, \frac{u_{3}}{0.4}, \frac{u_{4}}{0.2}\right\}\right), \\
& \left.\left(\left(e_{3}, r, 0\right),\left\{\frac{u_{1}}{0.5}, \frac{u_{2}}{0.4}, \frac{u_{3}}{0.6}, \frac{u_{4}}{0.5}\right\}\right)\right\} .
\end{aligned}
$$

Definition 19 The complement of a fuzzy soft expert set $(F, A)$ is denoted by $(F, A)^{c}$ and is defined by $\left.(F, A)^{c}=\left(F^{c},\right\rceil A\right)$ where $F^{c}: A \rightarrow I^{U}$ is a mapping given by

$$
F^{c}(\alpha)=c(F(\alpha)), \forall \alpha \in A,
$$

where $c$ is a fuzzy complement.

Example 4 Consider Example 1. By using the basic fuzzy complement, we have

$$
\begin{aligned}
& (F, Z)^{c}=\left\{\left(\left(e_{1}, p, 1\right),\left\{\frac{u_{1}}{0.7}, \frac{u_{2}}{0.5}, \frac{u_{3}}{0.3}, \frac{u_{4}}{0.9}\right\}\right),\left(\left(e_{1}, q, 1\right),\left\{\frac{u_{1}}{0.5}, \frac{u_{2}}{0.8}, \frac{u_{3}}{0.7}, \frac{0}{u_{4}}\right\}\right),\right. \\
& \left(\left(e_{1}, r, 1\right),\left\{\frac{u_{1}}{0.6}, \frac{u_{2}}{0.2}, \frac{u_{3}}{0.7}, \frac{u_{4}}{0.6}\right\}\right),\left(\left(e_{2}, p, 1\right),\left\{\frac{u_{1}}{0.7}, \frac{u_{2}}{0.8}, \frac{u_{3}}{0.5}, \frac{u_{4}}{0.4}\right\}\right), \\
& \left(\left(e_{2}, q, 1\right),\left\{\frac{u_{1}}{0.4}, \frac{u_{2}}{0.6}, \frac{u_{3}}{0.7}, \frac{u_{4}}{0.3}\right\}\right),\left(\left(e_{2}, r, 1\right),\left\{\frac{u_{1}}{0.9}, \frac{u_{2}}{0.7}, \frac{u_{3}}{0.3}, \frac{u_{4}}{0.6}\right\}\right), \\
& \left(\left(e_{3}, p, 1\right),\left\{\frac{u_{1}}{0.4}, \frac{u_{2}}{0.8}, \frac{u_{3}}{0.6}, \frac{u_{4}}{0.7}\right\}\right),\left(\left(e_{3}, q, 1\right),\left\{\frac{u_{1}}{0.5}, \frac{u_{2}}{0.7}, \frac{u_{3}}{0.5}, \frac{u_{4}}{0.3}\right\}\right), \\
& \left(\left(e_{3}, r, 1\right),\left\{\frac{u_{1}}{0.7}, \frac{u_{2}}{0.6}, \frac{u_{3}}{0.7}, \frac{u_{4}}{0.6}\right\}\right),\left(\left(e_{1}, p, 0\right),\left\{\frac{u_{1}}{0.4}, \frac{u_{2}}{0.6}, \frac{u_{3}}{0.6}, \frac{u_{4}}{0.4}\right\}\right), \\
& \left(\left(e_{1}, q, 0\right),\left\{\frac{u_{1}}{0.7}, \frac{u_{2}}{0.4}, \frac{u_{3}}{0.6}, \frac{1}{u_{4}}\right\}\right),\left(\left(e_{1}, r, 0\right),\left\{\frac{u_{1}}{0.5}, \frac{u_{2}}{0.9}, \frac{u_{3}}{0.3}, \frac{u_{4}}{0.5}\right\}\right), \\
& \left(\left(e_{2}, p, 0\right),\left\{\frac{u_{1}}{0.5}, \frac{u_{2}}{0.4}, \frac{u_{3}}{0.6}, \frac{u_{4}}{0.8}\right\}\right),\left(\left(e_{2}, q, 0\right),\left\{\frac{u_{1}}{0.8}, \frac{u_{2}}{0.6}, \frac{u_{3}}{0.5}, \frac{u_{4}}{0.9}\right\}\right) \text {, } \\
& \left(\left(e_{2}, r, 0\right),\left\{\frac{u_{1}}{0.3}, \frac{u_{2}}{0.5}, \frac{u_{3}}{0.8}, \frac{u_{4}}{0.5}\right\}\right),\left(\left(e_{3}, p, 0\right),\left\{\frac{u_{1}}{0.7}, \frac{u_{2}}{0.5}, \frac{u_{3}}{0.5}, \frac{u_{4}}{0.4}\right\}\right), \\
& \left.\left(\left(e_{3}, q, 0\right),\left\{\frac{u_{1}}{0.7}, \frac{u_{2}}{0.5}, \frac{u_{3}}{0.6}, \frac{u_{4}}{0.8}\right\}\right),\left(\left(e_{3}, r, 0\right),\left\{\frac{u_{1}}{0.5}, \frac{u_{2}}{0.6}, \frac{u_{3}}{0.4}, \frac{u_{4}}{0.5}\right\}\right)\right\} \text {. }
\end{aligned}
$$

Proposition 1 If $(F, A)$ is a fuzzy soft expert set over $U$, then

1. $\left((F, A)^{c}\right)^{c}=(F, A)$,

Proof From Definition 19 we have $(F, A)^{c}=\left(F^{c}, A\right)$ where $F^{c}(\alpha)=\overline{1}-F(\alpha), \forall \alpha \in A$. Now, $\left((F, A)^{c}\right)^{c}=\left(\left(F^{c}\right)^{c}, A\right)$ 
where $\left(F^{c}\right)^{c}(\alpha)=\overline{1}-(\overline{1}-F(\alpha)), \forall(\alpha) \in A$

$$
=F(\alpha), \forall \alpha \in A \text {. }
$$

\section{Union and Intersection}

In this section, we introduce the definitions of union and intersection of fuzzy soft expert sets, derive their properties, and give some examples.

Definition 20 The union of two fuzzy soft expert sets $(F, A)$ and $(G, B)$ over $U$, denoted by $(F, A) \tilde{\cup}(G, B)$, is the fuzzy soft expert set $(H, C)$ where $C=A \cup B$, and $\forall \varepsilon \in C$,

$$
H(\varepsilon)= \begin{cases}F(\varepsilon), & \text { if } \varepsilon \in A-B \\ G(\varepsilon), & \text { if } \varepsilon \in B-A \\ s(F(\varepsilon), G(\varepsilon)), & \text { if } \varepsilon \in A \bigcap B\end{cases}
$$

where $s$ is an s-norm.

Example 5 Consider Example 1. Let

$$
A=\left\{\left(e_{1}, p, 1\right),\left(e_{2}, p, 0\right),\left(e_{3}, p, 1\right),\left(e_{1}, q, 1\right),\left(e_{2}, q, 1\right),\left(e_{3}, q, 0\right),\left(e_{1}, r, 0\right),\left(e_{2}, r, 1\right),\left(e_{3}, r, 1\right)\right\}
$$

and

$$
B=\left\{\left(e_{1}, p, 1\right),\left(e_{2}, p, 0\right),\left(e_{3}, p, 0\right),\left(e_{1}, q, 1\right),\left(e_{2}, q, 1\right),\left(e_{3}, q, 1\right),\left(e_{1}, r, 0\right),\left(e_{2}, r, 1\right)\right\} .
$$

Suppose $(F, A)$ and $(G, B)$ are two fuzzy soft expert sets over $U$ such that

$$
\begin{aligned}
&(F, A)=\{\left(\left(e_{1}, p, 1\right),\left\{\frac{u_{1}}{0.3}, \frac{u_{2}}{0.5}, \frac{u_{3}}{0.5}, \frac{u_{4}}{0.6}\right\}\right),\left(\left(e_{1}, q, 1\right),\left\{\frac{u_{1}}{0.4}, \frac{u_{2}}{0.2}, \frac{u_{3}}{0.6}, \frac{u_{4}}{0.3}\right\}\right), \\
&\left(\left(e_{2}, q, 1\right),\left\{\frac{u_{1}}{0.1}, \frac{u_{2}}{0.2}, \frac{u_{3}}{0.7}, \frac{u_{4}}{0.4}\right\}\right),\left(\left(e_{2}, r, 1\right),\left\{\frac{u_{1}}{0.4}, \frac{u_{2}}{0.4}, \frac{u_{3}}{0.7}, \frac{u_{4}}{0.8}\right\}\right), \\
&\left(\left(e_{3}, p, 1\right),\left\{\frac{u_{1}}{0.6}, \frac{u_{2}}{0.3}, \frac{u_{3}}{0.4}, \frac{u_{4}}{0.2}\right\}\right),\left(\left(e_{3}, r, 1\right),\left\{\frac{u_{1}}{0.1}, \frac{u_{2}}{0.5}, \frac{u_{3}}{0.3}, \frac{u_{4}}{0.4}\right\}\right), \\
&\left(\left(e_{1}, r, 0\right),\left\{\frac{u_{1}}{0.5}, \frac{u_{2}}{0.2}, \frac{u_{3}}{0.8}, \frac{u_{4}}{0.3}\right\}\right),\left(\left(e_{2}, p, 0\right),\left\{\frac{u_{1}}{0.1}, \frac{u_{2}}{0.3}, \frac{u_{3}}{0.7}, \frac{u_{4}}{0.3}\right\}\right), \\
&(G, B)=\left\{\left(\left(e_{1}, p, 1\right),\left\{\frac{u_{1}}{0.2}, \frac{u_{2}}{0.1}, \frac{u_{3}}{0.6}, \frac{u_{4}}{0.8}\right\}\right),\left(\left(e_{1}, q, 1\right),\left\{\frac{u_{1}}{0.5}, \frac{u_{2}}{0.2}, \frac{u_{3}}{0.4}, \frac{u_{4}}{0.7}\right\}\right),\right. \\
&\left(\left(e_{2}, q, 1\right),\left\{\frac{u_{1}}{0.7}, \frac{u_{2}}{0.3}, \frac{u_{3}}{0.2}, \frac{u_{4}}{0.2}, \frac{u_{3}}{0.2}, \frac{u_{4}}{0.5}\right\}\right),\left(\left(e_{2}, r, 1\right),\left\{\frac{u_{1}}{0.3}, \frac{u_{2}}{0.5}, \frac{u_{3}}{0.5}, \frac{u_{4}}{0.6}\right\}\right), \\
&\left(\left(e_{1}, r, 0\right),\left\{\frac{u_{1}}{0.5}, \frac{u_{2}}{0.3}, \frac{u_{3}}{0.6}, \frac{u_{4}}{0.5}\right\}\right),\left(\left(e_{3}, p, 0\right),\left\{\frac{u_{1}}{0.1}, \frac{u_{2}}{0.8}, \frac{u_{3}}{0.2}, \frac{u_{4}}{0.9}\right\}\right), \\
&\left.\left(\left(e_{3}, q, 0\right),\left\{\frac{u_{1}}{0.6}, \frac{u_{2}}{0.5}, \frac{u_{3}}{0.3}, \frac{u_{4}}{0.2}\right\}\right),\left(\left(e_{2}, p, 0\right),\left\{\frac{u_{1}}{0.4}, \frac{u_{2}}{0.1}, \frac{u_{3}}{0.9}, \frac{u_{4}}{0.4}\right\}\right)\right\} .
\end{aligned}
$$

By using basic fuzzy union (maximum) we have $(F, A) \tilde{U}(G, B)=(H, C)$ where 


$$
\begin{aligned}
(H, C)=\left\{\left(\left(e_{1}, p, 1\right),\left\{\frac{u_{1}}{0.3}, \frac{u_{2}}{0.5}, \frac{u_{3}}{0.6}, \frac{u_{4}}{0.8}\right\}\right),\left(\left(e_{1}, q, 1\right),\left\{\frac{u_{1}}{0.5}, \frac{u_{2}}{0.2}, \frac{u_{3}}{0.6}, \frac{u_{4}}{0.7}\right\}\right),\right. \\
\left(\left(e_{2}, q, 1\right),\left\{\frac{u_{1}}{0.7}, \frac{u_{2}}{0.3}, \frac{u_{3}}{0.7}, \frac{u_{4}}{0.9}\right\}\right),\left(\left(e_{2}, r, 1\right),\left\{\frac{u_{1}}{0.4}, \frac{u_{2}}{0.5}, \frac{u_{3}}{0.7}, \frac{u_{4}}{0.8}\right\}\right), \\
\left(\left(e_{3}, p, 1\right),\left\{\frac{u_{1}}{0.6}, \frac{u_{2}}{0.3}, \frac{u_{3}}{0.4}, \frac{u_{4}}{0.2}\right\}\right),\left(\left(e_{3}, r, 1\right),\left\{\frac{u_{1}}{0.1}, \frac{u_{2}}{0.5}, \frac{u_{3}}{0.3}, \frac{u_{4}}{0.4}\right\}\right), \\
\left(\left(e_{1}, r, 0\right),\left\{\frac{u_{1}}{0.5}, \frac{u_{2}}{0.3}, \frac{u_{3}}{0.8}, \frac{u_{4}}{0.5}\right\}\right),\left(\left(e_{2}, p, 0\right),\left\{\frac{u_{1}}{0.4}, \frac{u_{2}}{0.3}, \frac{u_{3}}{0.9}, \frac{u_{4}}{0.4}\right\}\right), \\
\left.\left(\left(e_{3}, q, 0\right),\left\{\frac{u_{1}}{0.6}, \frac{u_{2}}{0.5}, \frac{u_{3}}{0.3}, \frac{u_{4}}{0.5}\right\}\right),\left(\left(e_{3}, p, 0\right),\left\{\frac{u_{1}}{0.1}, \frac{u_{2}}{0.8}, \frac{u_{3}}{0.2}, \frac{u_{4}}{0.9}\right\}\right)\right\} .
\end{aligned}
$$

Proposition 2 If $(F, A),(G, B)$ and $(H, C)$ are three fuzzy soft expert sets over $U$, then

1. $(F, A) \tilde{U}((G, B) \tilde{\cup}(H, C))=((F, A) \tilde{U}(G, B)) \tilde{U}(H, C)$,

2. $(F, A) \tilde{U}(F, A) \subseteq(F, A)$.

Proof $a$. We want to prove that $(F, A) \tilde{U}((G, B) \tilde{U}(H, C))=((F, A) \tilde{U}(G, B)) \tilde{U}(H, C)$.

By using definition 20 we have

$$
((G, B) \tilde{\cup}(H, C))= \begin{cases}G(\varepsilon), & \text { if } \varepsilon \in B-C \\ H(\varepsilon), & \text { if } \varepsilon \in C-B \\ s(G(\varepsilon), H(\varepsilon)), & \text { if } \varepsilon \in B \cap C\end{cases}
$$

We consider the case when $\varepsilon \in B \bigcap C$ as the other cases are trivial, then we have

$$
(G, B) \tilde{U}(H, C)=(s(G(\varepsilon), H(\varepsilon)), B \cup C) .
$$

We also consider her the case when $\varepsilon \in A$ as the other cases are trivial, then we have

$$
\begin{aligned}
& (F, A) \tilde{\cup}((G, B) \tilde{\cup}(H, C)) \\
& =(s(F(\varepsilon), s(G(\varepsilon), H(\varepsilon))), A \cup(B \cup C)) \\
& =(s(s(F(\varepsilon), G(\varepsilon)) \cup H(\varepsilon)),(A \cup B) \cup C) \\
& =((F, A) \tilde{\cup}(G, B)) \tilde{\cup}(H, C)
\end{aligned}
$$

b. The proof is straightforward.

Definition 21 The intersection of two fuzzy soft expert sets $(F, A)$ and $(G, B)$ over $U$, denoted by $(F, A) \tilde{\bigcap}(G, B)$, is the fuzzy soft expert set $(H, C)$ where $C=A \cup B$, and $\forall \varepsilon \in C$,

$$
H(\varepsilon)= \begin{cases}F(\varepsilon), & \text { if } \varepsilon \in A-B \\ G(\varepsilon), & \text { if } \varepsilon \in B-A \\ t(F(\varepsilon), G(\varepsilon)), & \text { if } \varepsilon \in A \cap B\end{cases}
$$

where $t$ is a $t$-norm.

Example 6 Consider Example 5. By using basic fuzzy intersection (minimum) we have $(F, A) \tilde{\cap}(G, B)=(H, C)$ where 


$$
\begin{aligned}
(H, C)=\{ & \left(\left(e_{1}, p, 1\right),\left\{\frac{u_{1}}{0.2}, \frac{u_{2}}{0.1}, \frac{u_{3}}{0.5}, \frac{u_{4}}{0.6}\right\}\right),\left(\left(e_{1}, q, 1\right),\left\{\frac{u_{1}}{0.4}, \frac{u_{2}}{0.2}, \frac{u_{3}}{0.4}, \frac{u_{4}}{0.3}\right\}\right), \\
& \left(\left(e_{2}, q, 1\right),\left\{\frac{u_{1}}{0.1}, \frac{u_{2}}{0.2}, \frac{u_{3}}{0.2}, \frac{u_{4}}{0.4}\right\}\right),\left(\left(e_{2}, r, 1\right),\left\{\frac{u_{1}}{0.3}, \frac{u_{2}}{0.4}, \frac{u_{3}}{0.5}, \frac{u_{4}}{0.6}\right\}\right), \\
& \left(\left(e_{3}, p, 1\right),\left\{\frac{u_{1}}{0.6}, \frac{u_{2}}{0.3}, \frac{u_{3}}{0.4}, \frac{u_{4}}{0.2}\right\}\right),\left(\left(e_{3}, r, 1\right),\left\{\frac{u_{1}}{0.1}, \frac{u_{2}}{0.5}, \frac{u_{3}}{0.3}, \frac{u_{4}}{0.4}\right\}\right), \\
& \left(\left(e_{1}, r, 0\right),\left\{\frac{u_{1}}{0.5}, \frac{u_{2}}{0.2}, \frac{u_{3}}{0.6}, \frac{u_{4}}{0.3}\right\}\right),\left(\left(e_{2}, p, 0\right),\left\{\frac{u_{1}}{0.1}, \frac{u_{2}}{0.1}, \frac{u_{3}}{0.7}, \frac{u_{4}}{0.3}\right\}\right), \\
& \left.\left(\left(e_{3}, q, 0\right),\left\{\frac{u_{1}}{0.5}, \frac{u_{2}}{0.2}, \frac{u_{3}}{0.2}, \frac{u_{4}}{0.2}\right\}\right),\left(\left(e_{3}, p, 0\right),\left\{\frac{u_{1}}{0.1}, \frac{u_{2}}{0.8}, \frac{u_{3}}{0.2}, \frac{u_{4}}{0.9}\right\}\right)\right\} .
\end{aligned}
$$

Proposition 3 If $(F, A),(G, B)$ and $(H, C)$ are three fuzzy soft expert sets over $U$, then

1. $(F, A) \tilde{\cap}((G, B) \tilde{\cap}(H, C))=((F, A) \tilde{\cap}(G, B)) \tilde{\cap}(H, C)$,

2. $(F, A) \tilde{\cap}(F, A) \subseteq(F, A)$.

Proof $a$. We want to prove that $(F, A) \tilde{\cap}((G, B) \tilde{\cap}(H, C))=((F, A) \tilde{\cap}(G, B)) \tilde{\cap}(H, C)$.

By using definition 21 we have

$$
((G, B) \tilde{U}(H, C))= \begin{cases}G(\varepsilon), & \text { if } \varepsilon \in B-C \\ H(\varepsilon), & \text { if } \varepsilon \in C-B \\ t(G(\varepsilon), H(\varepsilon)), & \text { if } \varepsilon \in B \cap C .\end{cases}
$$

We consider the case when $\varepsilon \in B \cap C$ as the other cases are trivial, then we have

$$
(G, B) \tilde{\cap}(H, C)=(t(G(\varepsilon), H(\varepsilon)), B \cup C) .
$$

We also consider her the case when $\varepsilon \in A$ as the other cases are trivial, then we have

$$
\begin{aligned}
& (F, A) \tilde{\cap}((G, B) \tilde{\cap}(H, C)) \\
& =(t(F(\varepsilon), t(G(\varepsilon), H(\varepsilon))), A \cup(B \cup C)) \\
& =(t(t(F(\varepsilon), G(\varepsilon)) \cap H(\varepsilon)),(A \cup B) \cup C) \\
& =((F, A) \tilde{\cap}(G, B)) \tilde{\cap}(H, C)
\end{aligned}
$$

$b$. The proof is straightforward.

Proposition 4 If $(F, A),(G, B)$ and $(H, C)$ are three fuzzy soft expert sets over $U$, then

1. $(F, A) \tilde{U}((G, B) \tilde{\cap}(H, C))=((F, A) \tilde{U}(G, B)) \tilde{\cap}((F, A) \tilde{U}(H, C))$,

2. $(F, A) \tilde{ก}((G, B) \tilde{U}(H, C))=((F, A) \tilde{\cap}(G, B)) \tilde{U}((F, A) \tilde{\cap}(H, C))$.

Proof $a$. We want to prove that $(F, A) \tilde{U}((G, B) \tilde{\cap}(H, C))=((F, A) \tilde{U}(G, B)) \tilde{\cap}((F, A) \tilde{\cup}(H, C))$, By using definitions 20 and 21 we have

$$
((G, B) \tilde{\cap}(H, C))= \begin{cases}G(\varepsilon), & \text { if } \varepsilon \in B-C \\ H(\varepsilon), & \text { if } \varepsilon \in C-B \\ t(G(\varepsilon), H(\varepsilon)), & \text { if } \varepsilon \in B \cap C .\end{cases}
$$

We consider the case when $\varepsilon \in B \cap C$ as the other cases are trivial, then we have

$$
(G, B) \tilde{\cap}(H, C)=(t(G(\varepsilon), H(\varepsilon)), B \cup C) .
$$


We also consider her the case when $\varepsilon \in A$ as the other cases are trivial, then we have

$$
\begin{aligned}
& (F, A) \tilde{U}((G, B) \tilde{\cap}(H, C)) \\
& =(s(F(\varepsilon), t(G(\varepsilon), H(\varepsilon))), A \cup(B \cup C)) . \\
& =t(s(F(\varepsilon), G(\varepsilon)), s(F(\varepsilon) \cup H(\varepsilon))) \\
& =((F, A) \tilde{\cup}(G, B)) \tilde{\cap}((F, A) \tilde{\cup}(H, C))
\end{aligned}
$$

b. We want to prove that $(F, A) \tilde{\cap}((G, B) \tilde{U}(H, C))=((F, A) \tilde{\cap}(G, B)) \tilde{U}((F, A) \tilde{U}(H, C))$,

By using definitions 20 and 21 we have

$$
((G, B) \tilde{U}(H, C))= \begin{cases}G(\varepsilon), & \text { if } \varepsilon \in B-C \\ H(\varepsilon), & \text { if } \varepsilon \in C-B \\ s(G(\varepsilon), H(\varepsilon)), & \text { if } \varepsilon \in B \cap C .\end{cases}
$$

We consider the case when $\varepsilon \in B \cap C$ as the other cases are trivial, then we have

$$
(G, B) \tilde{U}(H, C)=(s(G(\varepsilon), H(\varepsilon)), B \cup C) .
$$

We also consider her the case when $\varepsilon \in A$ as the other cases are trivial, then we have

$$
\begin{aligned}
& (F, A) \tilde{\cap}((G, B) \tilde{U}(H, C))=(t(F(\varepsilon), s(G(\varepsilon), H(\varepsilon))), A \cup(B \cup C)) \\
& =s(t(F(\varepsilon), G(\varepsilon)), t(F(\varepsilon) \cup H(\varepsilon)))=((F, A) \tilde{\cap}(G, B)) \tilde{U}((F, A) \tilde{U}(H, C))
\end{aligned} .
$$

\section{AND and OR Operations}

In this section, we introduce the definitions of AND and OR operations for fuzzy soft expert sets, derive their properties, and give some examples.

Definition 22 If $(F, A)$ and $(G, B)$ are two fuzzy soft expert sets over $U$ then " $(F, A)$ AND $(G, B)$ " denoted by $(F, A) \wedge(G, B)$ is defined by

$$
(F, A) \wedge(G, B)=(H, A \times B)
$$

such that $H(\alpha, \beta)=t(F(\alpha), G(\beta)), \forall(\alpha, \beta) \in A \times B$, where $t$ is a $t$-norm.

Example 7 Consider Example 1. Let

$$
\begin{gathered}
A=\left\{\left(e_{1}, p, 1\right),\left(e_{2}, p, 0\right),\left(e_{1}, r, 0\right),\left(e_{2}, r, 1\right)\right\} \text { and } \\
B=\left\{\left(e_{1}, p, 1\right),\left(e_{1}, r, 0\right),\left(e_{2}, r, 1\right)\right\} .
\end{gathered}
$$

Suppose $(F, A)$ and $(G, B)$ are two fuzzy soft expert sets over $U$ such that

$$
\begin{aligned}
&(F, A)=\{\left(\left(e_{1}, p, 1\right),\left\{\frac{u_{1}}{0.5}, \frac{u_{2}}{0.2}, \frac{u_{3}}{0.2}, \frac{u_{4}}{0.2}\right\}\right),\left(\left(e_{1}, r, 0\right),\left\{\frac{u_{1}}{0.3}, \frac{u_{2}}{0.1}, \frac{u_{3}}{0.5}, \frac{u_{4}}{0.4}\right\}\right), \\
&\left.\left(\left(e_{3}, q, 0\right),\left\{\frac{u_{1}}{0.2}, \frac{u_{2}}{0.5}, \frac{u_{3}}{0.6}, \frac{u_{4}}{0.8}\right\}\right),\left(\left(e_{2}, r, 1\right),\left\{\frac{u_{1}}{0.6}, \frac{u_{2}}{0.4}, \frac{u_{3}}{0.2}, \frac{u_{4}}{0.3}\right\}\right)\right\} \\
&(G, B)=\left\{\left(\left(e_{1}, p, 1\right),\left\{\frac{u_{1}}{0.2}, \frac{u_{2}}{0.6}, \frac{u_{3}}{0.4}, \frac{u_{4}}{0.5}\right\}\right),\left(\left(e_{1}, r, 0\right),\left\{\frac{u_{1}}{0.7}, \frac{u_{2}}{0.4}, \frac{u_{3}}{0.3}, \frac{u_{4}}{0.5}\right\}\right),\right. \\
&\left.\left(\left(e_{2}, r, 1\right),\left\{\frac{u_{1}}{0.4}, \frac{u_{2}}{0.6}, \frac{u_{3}}{0.7}, \frac{u_{4}}{0.8}\right\}\right)\right\} .
\end{aligned}
$$

By using basic fuzzy intersection (minimum) we have $(F, A) \wedge(G, B)=(H, A \times B)$ where 


$$
\begin{aligned}
(H, A \times B)=\{ & \left(\left(\left(e_{1}, p, 1\right),\left(e_{1}, p, 1\right)\right),\left\{\frac{u_{1}}{0.2}, \frac{u_{2}}{0.2}, \frac{u_{3}}{0.2}, \frac{u_{4}}{0.2}\right\}\right),\left(\left(\left(e_{1}, p, 1\right),\left(e_{1}, r, 0\right)\right),\left\{\frac{u_{1}}{0.5}, \frac{u_{2}}{0.2}, \frac{u_{3}}{0.2}, \frac{u_{4}}{0.2}\right\}\right), \\
& \left(\left(\left(e_{1}, p, 1\right),\left(e_{2}, r, 1\right)\right),\left\{\frac{u_{1}}{0.4}, \frac{u_{2}}{0.2}, \frac{u_{3}}{0.2}, \frac{u_{4}}{0.2}\right\}\right),\left(\left(\left(e_{1}, r, 0\right),\left(e_{1}, p, 1\right)\right),\left\{\frac{u_{1}}{0.2}, \frac{u_{2}}{0.1}, \frac{u_{3}}{0.4}, \frac{u_{4}}{0.4}\right\}\right), \\
& \left(\left(\left(e_{1}, r, 0\right),\left(e_{1}, r, 0\right)\right),\left\{\frac{u_{1}}{0.3}, \frac{u_{2}}{0.1}, \frac{u_{3}}{0.3}, \frac{u_{4}}{0.4}\right\}\right),\left(\left(\left(e_{1}, r, 0\right),\left(e_{2}, r, 1\right)\right),\left\{\frac{u_{1}}{0.3}, \frac{u_{2}}{0.1}, \frac{u_{3}}{0.5}, \frac{u_{4}}{0.4}\right\}\right), \\
& \left(\left(\left(e_{3}, q, 0\right),\left(e_{1}, p, 1\right)\right),\left\{\frac{u_{1}}{0.2}, \frac{u_{2}}{0.5}, \frac{u_{3}}{0.4}, \frac{u_{4}}{0.5}\right\}\right),\left(\left(\left(e_{3}, q, 0\right),\left(e_{1}, r, 0\right)\right),\left\{\frac{u_{1}}{0.2}, \frac{u_{2}}{0.4}, \frac{u_{3}}{0.3}, \frac{u_{4}}{0.5}\right\}\right), \\
& \left(\left(\left(e_{3}, q, 0\right),\left(e_{2}, r, 1\right)\right),\left\{\frac{u_{1}}{0.2}, \frac{u_{2}}{0.5}, \frac{u_{3}}{0.6}, \frac{u_{4}}{0.8}\right\}\right),\left(\left(\left(e_{2}, r, 1\right),\left(e_{1}, p, 1\right)\right),\left\{\frac{u_{1}}{0.2}, \frac{u_{2}}{0.4}, \frac{u_{3}}{0.2}, \frac{u_{4}}{0.3}\right\}\right), \\
& \left.\left(\left(\left(e_{2}, r, 1\right),\left(e_{1}, r, 0\right)\right),\left\{\frac{u_{1}}{0.6}, \frac{u_{2}}{0.4}, \frac{u_{3}}{0.2}, \frac{u_{4}}{0.3}\right\}\right),\left(\left(\left(e_{2}, r, 1\right),\left(e_{2}, r, 1\right)\right),\left\{\frac{u_{1}}{0.4}, \frac{u_{2}}{0.4}, \frac{u_{3}}{0.2}, \frac{u_{4}}{0.3}\right\}\right)\right\} .
\end{aligned}
$$

Definition 23 If $(F, A)$ and $(G, B)$ are two fuzzy soft expert sets over $U$ then " $(F, A)$ OR $(G, B)$ ” denoted by $(F, A) \vee(G, B)$ is defined by

$$
(F, A) \vee(G, B)=(H, A \times B)
$$

such that $H(\alpha, \beta)=s(F(\alpha), G(\beta)), \forall(\alpha, \beta) \in A \times B$, where $s$ is an $s$-norm.

Example 8 Consider Example 7. By using basic fuzzy union (maximum) we have $(F, A) \vee(G, B)=(H, A \times B)$ where

$$
\begin{aligned}
(H, A \times B)=\{ & \left(\left(\left(e_{1}, p, 1\right),\left(e_{1}, p, 1\right)\right),\left\{\frac{u_{1}}{0.5}, \frac{u_{2}}{0.5}, \frac{u_{3}}{0.6}, \frac{u_{4}}{0.8}\right\}\right),\left(\left(\left(e_{1}, p, 1\right),\left(e_{1}, r, 0\right)\right),\left\{\frac{u_{1}}{0.7}, \frac{u_{2}}{0.4}, \frac{u_{3}}{0.3}, \frac{u_{4}}{0.5}\right\}\right), \\
& \left(\left(\left(e_{1}, p, 1\right),\left(e_{2}, r, 1\right)\right),\left\{\frac{u_{1}}{0.5}, \frac{u_{2}}{0.6}, \frac{u_{3}}{0.7}, \frac{u_{4}}{0.8}\right\}\right),\left(\left(\left(e_{1}, r, 0\right),\left(e_{1}, p, 1\right)\right),\left\{\frac{u_{1}}{0.3}, \frac{u_{2}}{0.6}, \frac{u_{3}}{0.5}, \frac{u_{4}}{0.5}\right\}\right), \\
& \left(\left(\left(e_{1}, r, 0\right),\left(e_{1}, r, 0\right)\right),\left\{\frac{u_{1}}{0.3}, \frac{u_{2}}{0.1}, \frac{u_{3}}{0.3}, \frac{u_{4}}{0.4}\right\}\right),\left(\left(\left(e_{1}, r, 0\right),\left(e_{2}, r, 1\right)\right),\left\{\frac{u_{1}}{0.4}, \frac{u_{2}}{0.6}, \frac{u_{3}}{0.7}, \frac{u_{4}}{0.8}\right\}\right), \\
& \left(\left(\left(e_{3}, q, 0\right),\left(e_{1}, p, 1\right)\right),\left\{\frac{u_{1}}{0.2}, \frac{u_{2}}{0.6}, \frac{u_{3}}{0.6}, \frac{u_{4}}{0.8}\right\}\right),\left(\left(\left(e_{3}, q, 0\right),\left(e_{1}, r, 0\right)\right),\left\{\frac{u_{1}}{0.7}, \frac{u_{2}}{0.6}, \frac{u_{3}}{0.5}, \frac{u_{4}}{0.8}\right\}\right), \\
& \left(\left(\left(e_{3}, q, 0\right),\left(e_{2}, r, 1\right)\right),\left\{\frac{u_{1}}{0.4}, \frac{u_{2}}{0.6}, \frac{u_{3}}{0.7}, \frac{u_{4}}{0.8}\right\}\right),\left(\left(\left(e_{2}, r, 1\right),\left(e_{1}, p, 1\right)\right),\left\{\frac{u_{1}}{0.6}, \frac{u_{2}}{0.6}, \frac{u_{3}}{0.4}, \frac{u_{4}}{0.5}\right\}\right), \\
& \left.\left(\left(\left(e_{2}, r, 1\right),\left(e_{1}, r, 0\right)\right),\left\{\frac{u_{1}}{0.7}, \frac{u_{2}}{0.4}, \frac{u_{3}}{0.3}, \frac{u_{4}}{0.5}\right\}\right),\left(\left(\left(e_{2}, r, 1\right),\left(e_{2}, r, 1\right)\right),\left\{\frac{u_{1}}{0.6}, \frac{u_{2}}{0.6}, \frac{u_{3}}{0.7}, \frac{u_{4}}{0.8}\right\}\right)\right\} .
\end{aligned}
$$

Proposition 5 If $(F, A)$ and $(G, B)$ are two fuzzy soft expert sets over $U$, then [a.]

1. $((F, A) \wedge(G, B))^{c}=(F, A)^{c} \vee(G, B)^{c}$

2. $((F, A) \vee(G, B))^{c}=(F, A)^{c} \wedge(G, B)^{c}$

Proof $a$. Suppose that $(F, A) \wedge(G, B)=(O, A \times B)$.

Therefore, $((F, A) \wedge(G, B))^{c}=(O, A \times B)^{c}=\left(O^{c},(A \times B)\right)$. Now,

$$
((F, A) \vee(G, B))^{c}=\left(\left(F^{c}, A\right) \vee\left(G^{c}, B\right)\right)=(J,(A \times B)),
$$

where $J(x, y)=t(c(F(\alpha)), c(G(\beta)))$.

Now, take $(\alpha, \beta) \in(A \times B)$.

Therefore, 


$$
\begin{aligned}
O^{c}(\alpha, \beta) & =\overline{1}-O(\alpha, \beta)=\overline{1}-[F(\alpha) \cup G(\beta)] \\
& =[\overline{1}-F(\alpha)] \cap[\overline{1}-G(\beta)], \\
& =t(c(F(\alpha)), c(G(\beta)))=J(\alpha, \beta)
\end{aligned},
$$

Then $O^{c}$ and $J$ are the same. Hence, proved.

$b$. Suppose that $(F, A) \vee(G, B)=(O, A \times B)$.

Therefore, $((F, A) \vee(G, B))^{c}=(O, A \times B)^{c}=\left(O^{c},(A \times B)\right)$. Now,

$((F, A) \wedge(G, B))^{c}=\left(\left(F^{c}, A\right) \wedge\left(G^{c}, B\right)\right)=(J,(A \times B))$,

where $J(x, y)=s(c(F(\alpha)), c(G(\beta)))$.

Now, take $(\alpha, \beta) \in(A \times B)$.

Therefore,

$$
\begin{aligned}
O^{c}(\alpha, \beta) & =\overline{1}-O(\alpha, \beta)=\overline{1}-[F(\alpha) \cap G(\beta)] \\
& =[\overline{1}-F(\alpha)] \cup[\overline{1}-G(\beta)], \\
& =s(c(F(\alpha)), c(G(\beta)))=J(\alpha, \beta)
\end{aligned}
$$

Then $O^{c}$ and $J$ are the same. Hence, proved.

Proposition 6 If $(F, A),(G, B)$ and $(H, C)$ are three fuzzy soft expert sets over $U$, then

1. $(F, A) \wedge((G, B) \wedge(H, C))=((F, A) \wedge(G, B)) \wedge(H, C)$,

2. $(F, A) \vee((G, B) \vee(H, C))=((F, A) \vee(G, B)) \vee(H, C)$,

3. $(F, A) \vee((G, B) \wedge(H, C))=((F, A) \vee(G, B)) \wedge((F, A) \vee(H, C))$,

4. $(F, A) \wedge((G, B) \vee(H, C))=((F, A) \wedge(G, B)) \vee((F, A) \wedge(H, C))$.

Proof We give the proofs of $a$ and $b$. [a.]

1. Suppose that $(G, B) \wedge(H, C)=t(G(\alpha), H(\beta)), \forall(\alpha, \beta) \in B \times C$.

Therefore

$$
\begin{aligned}
& (F, A) \wedge((G, B) \wedge(H, C))=t(F(\gamma), t(G(\alpha), H(\beta))), \quad \forall(\gamma,(\alpha, \beta)) \in A \times(B \times C) . \\
& =t(t(F(\gamma), G(\alpha)), H(\beta)), \quad \forall((\gamma, \alpha), \beta) \in(A \times B) \times C . \\
& =((F, A) \wedge(G, B)) \wedge(H, C) .
\end{aligned}
$$

2. Suppose that $(G, B) \vee(H, C)=s(G(\alpha), H(\beta)), \forall(\alpha, \beta) \in B \times C$.

Therefore

$$
\begin{aligned}
& (F, A) \vee((G, B) \vee(H, C))=s(F(\gamma), s(G(\alpha), H(\beta))), \quad \forall(\gamma,(\alpha, \beta)) \in A \times(B \times C) . \\
& =s(s(F(\gamma), G(\alpha)), H(\beta)), \quad \forall((\gamma, \alpha), \beta) \in(A \times B) \times C . \\
& =((F, A) \vee(G, B)) \vee(H, C) .
\end{aligned}
$$

Remark The commutativity do not hold in AND and OR operations since $A \times B \neq B \times A$.

\section{An Application of Fuzzy Soft Expert Set in Decision Making}

In this section, we present an application of fuzzy soft expert set theory in a decision making problem. Assume that a company wants to fill a position. There are four candidates who form the universe $U=\left\{u_{1}, u_{2}, u_{3}, u_{4}\right\}$, the hiring committee considers a set of parameters, $E=\left\{e_{1}, e_{2}, e_{3}\right\}$, the parameters $e_{i}(i=1,2,3)$ stand for "experience", "computer knowledge" and "good speaking” respectively. Let $X=\{p, q, r\}$ be a set of experts (Committee members). After a serious discussion the committee constructs the following fuzzy soft expert set 


$$
\begin{aligned}
(F, Z)=\{ & \left(\left(e_{1}, p, 1\right),\left\{\frac{u_{1}}{0.3}, \frac{u_{2}}{0.5}, \frac{u_{3}}{0.7}, \frac{u_{4}}{0.1}\right\}\right),\left(\left(e_{1}, q, 1\right),\left\{\frac{u_{1}}{0.5}, \frac{u_{2}}{0.2}, \frac{u_{3}}{0.3}, \frac{1}{u_{4}}\right\}\right), \\
& \left(\left(e_{1}, r, 1\right),\left\{\frac{u_{1}}{0.4}, \frac{u_{2}}{0.8}, \frac{u_{3}}{0.3}, \frac{u_{4}}{0.4}\right\}\right),\left(\left(e_{2}, p, 1\right),\left\{\frac{u_{1}}{0.3}, \frac{u_{2}}{0.2}, \frac{u_{3}}{0.5}, \frac{u_{4}}{0.6}\right\}\right), \\
& \left(\left(e_{2}, q, 1\right),\left\{\frac{u_{1}}{0.6}, \frac{u_{2}}{0.4}, \frac{u_{3}}{0.3}, \frac{u_{4}}{0.7}\right\}\right),\left(\left(e_{2}, r, 1\right),\left\{\frac{u_{1}}{0.1}, \frac{u_{2}}{0.3}, \frac{u_{3}}{0.7}, \frac{u_{4}}{0.4}\right\}\right), \\
& \left(\left(e_{3}, p, 1\right),\left\{\frac{u_{1}}{0.6}, \frac{u_{2}}{0.2}, \frac{u_{3}}{0.4}, \frac{u_{4}}{0.3}\right\}\right),\left(\left(e_{3}, q, 1\right),\left\{\frac{u_{1}}{0.5}, \frac{u_{2}}{0.3}, \frac{u_{3}}{0.5}, \frac{u_{4}}{0.7}\right\}\right), \\
& \left(\left(e_{3}, r, 1\right),\left\{\frac{u_{1}}{0.3}, \frac{u_{2}}{0.4}, \frac{u_{3}}{0.3}, \frac{u_{4}}{0.4}\right\}\right),\left(\left(e_{1}, p, 0\right),\left\{\frac{u_{1}}{0.6}, \frac{u_{2}}{0.4}, \frac{u_{3}}{0.4}, \frac{u_{4}}{0.6}\right\}\right), \\
& \left(\left(e_{1}, q, 0\right),\left\{\frac{u_{1}}{0.3}, \frac{u_{2}}{0.6}, \frac{u_{3}}{0.4}, \frac{0}{u_{4}}\right\}\right),\left(\left(e_{1}, r, 0\right),\left\{\frac{u_{1}}{0.5}, \frac{u_{2}}{0.1}, \frac{u_{3}}{0.7}, \frac{u_{4}}{0.5}\right\}\right), \\
& \left(\left(e_{2}, p, 0\right),\left\{\frac{u_{1}}{0.5}, \frac{u_{2}}{0.6}, \frac{u_{3}}{0.4}, \frac{u_{4}}{0.2}\right\}\right),\left(\left(e_{2}, q, 0\right),\left\{\frac{u_{1}}{0.2}, \frac{u_{2}}{0.4}, \frac{u_{3}}{0.5}, \frac{u_{4}}{0.1}\right\}\right), \\
& \left(\left(e_{2}, r, 0\right),\left\{\frac{u_{1}}{0.7}, \frac{u_{2}}{0.5}, \frac{u_{3}}{0.2}, \frac{u_{4}}{0.5}\right\}\right),\left(\left(e_{3}, p, 0\right),\left\{\frac{u_{1}}{0.3}, \frac{u_{2}}{0.5}, \frac{u_{3}}{0.5}, \frac{u_{4}}{0.6}\right\}\right), \\
& \left.\left(\left(e_{3}, q, 0\right),\left\{\frac{u_{1}}{0.3}, \frac{u_{2}}{0.5}, \frac{u_{3}}{0.4}, \frac{u_{4}}{0.2}\right\}\right),\left(\left(e_{3}, r, 0\right),\left\{\frac{u_{1}}{0.5}, \frac{u_{2}}{0.4}, \frac{u_{3}}{0.6}, \frac{u_{4}}{0.5}\right\}\right)\right\} .
\end{aligned}
$$

In Table 1 and Table 2 we present the agree-fuzzy soft expert set and disagree-fuzzy soft expert set respectively.

The following algorithm may be followed by the company to fill the position.

1. Input the fuzzy soft expert set $(F, Z)$.

2. Find an agree-fuzzy soft expert set and a disagree-fuzzy soft expert set.

3. Find $c_{j}=\sum_{i} u_{i j}$ for agree-fuzzy soft expert set.

4. Find $k_{j}=\sum_{i} u_{i j}$ for disagree-fuzzy soft expert set.

5. Find $s_{j}=c_{j}-k_{j}$.

6. Find $m$, for which $s_{m}=\max s_{j}$. Then $s_{m}$ is the optimal choice object. If $m$ has more than one value, then any one of them could be chosen by the company using its option.

\section{Table 1. Agree-fuzzy soft expert set.}

\begin{tabular}{ccccc}
\hline$U$ & $u_{1}$ & $u_{2}$ & $u_{3}$ & $u_{4}$ \\
\hline$\left(e_{1}, p\right)$ & 0.3 & 0.5 & 0.7 & 0.1 \\
$\left(e_{2}, p\right)$ & 0.3 & 0.2 & 0.5 & 0.6 \\
$\left(e_{3}, p\right)$ & 0.6 & 0.2 & 0.4 & 0.3 \\
$\left(e_{1}, q\right)$ & 0.5 & 0.2 & 0.3 & 1 \\
$\left(e_{2}, q\right)$ & 0.6 & 0.4 & 0.3 & 0.7 \\
$\left(e_{3}, q\right)$ & 0.5 & 0.3 & 0.5 & 0.7 \\
$\left(e_{1}, r\right)$ & 0.4 & 0.8 & 0.3 & 0.4 \\
$\left(e_{2}, r\right)$ & 0.1 & 0.3 & 0.7 & 0.4 \\
$\left(e_{3}, r\right)$ & 0.3 & 0.4 & 0.3 & 0.4 \\
$c_{j}=\sum_{i} u_{i j}$ & $c_{1}=3.6$ & $c_{2}=3.3$ & $c_{3}=4$ & $c_{4}=4.6$ \\
\hline
\end{tabular}


Table 2. Disagree-fuzzy soft expert set.

\begin{tabular}{ccccc}
\hline$U$ & $u_{1}$ & $u_{2}$ & $u_{3}$ & $u_{4}$ \\
\hline$\left(e_{1}, p\right)$ & 0.6 & 0.4 & 0.4 & 0.6 \\
$\left(e_{2}, p\right)$ & 0.5 & 0.6 & 0.4 & 0.2 \\
$\left(e_{3}, p\right)$ & 0.3 & 0.5 & 0.5 & 0.6 \\
$\left(e_{1}, q\right)$ & 0.3 & 0.6 & 0.4 & 0 \\
$\left(e_{2}, q\right)$ & 0.2 & 0.4 & 0.5 & 0.1 \\
$\left(e_{3}, q\right)$ & 0.3 & 0.5 & 0.4 & 0.2 \\
$\left(e_{1}, r\right)$ & 0.5 & 0.1 & 0.7 & 0.5 \\
$\left(e_{2}, r\right)$ & 0.7 & 0.5 & 0.2 & 0.5 \\
$\left(e_{3}, r\right)$ & 0.5 & 0.4 & 0.6 & 0.5 \\
$k_{j}=\sum_{i} u_{i j}$ & $k_{1}=3.9$ & $k_{2}=4$ & $k_{3}=4.1$ & $k_{4}=3.2$
\end{tabular}

Now we use this algorithm to find the best choice for the company to fill the position. From Table 1 and Table 2, we have the following in Table 3:

Then $\max s_{j}=s_{4}$, so the committee will choose candidate 4 for the job.

\section{Mapping on Fuzzy Soft Expert Classes}

In this section, we introduce the notion of mapping on fuzzy soft expert classes. fuzzy soft expert classes are collections of fuzzy soft expert sets. We also define and study the properties of fuzzy soft expert images and fuzzy soft expert inverse images of fuzzy soft expert sets, and support them with example and theorems.

Definition 24 Let $U$ be a universe, $E$ a set of parameters, $X$ a set of experts (agents), and $O=\{1=$ agree, $0=$ disagree $\}$ a set of opinions. Let $Z=E \times X \times O$. Then the collection of all fuzzy soft expert

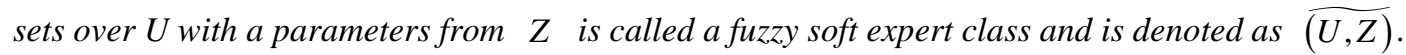

Definition 25 Let $\overline{(U, Z)}$ and $\overline{\left(Y, Z^{\prime}\right)}$ be fuzzy soft expert classes. Let $r: U \rightarrow Y$ and $s: Z \rightarrow Z^{\prime}$ be mappings. Then a mapping $f: \overline{(U, Z)} \rightarrow \overline{\left(Y, Z^{\prime}\right)}$ is defined as follows:

For a fuzzy soft expert set $(F, A)$ in $\overline{(U, Z)}, f(F, A)$ is a fuzzy soft expert in $\overline{\left(Y, Z^{\prime}\right)}$ obtained as follows:

$$
f(F, A)(\beta)(y)=\left\{\begin{array}{lc}
\bigvee_{x \in r^{-1}(y)}\left(\bigvee_{\alpha} F(\alpha)\right) & \text { if } r^{-1}(y) \text { and } s^{-1}(\beta) \cap A \neq \varnothing, \\
0 & \text { otherwise. }
\end{array}\right.
$$

For $\beta \in s(Z) \subseteq Z^{\prime}, \quad y \in Y$ and $\forall \alpha \in s^{-1}(\beta) \cap A . f(F, A)$ is called a fuzzy soft expert image of the fuzzy soft expert set $(F, A)$.

Definition 26 Let $\overline{(U, Z)}$ and $\overline{\left(Y, Z^{\prime}\right)}$ be fuzzy soft expert classes. Let $r: U \rightarrow Y$ and $s: Z \rightarrow Z^{\prime}$ be mappings. Then a mapping $f^{-1}: \overline{\left(Y, Z^{\prime}\right)} \rightarrow \overline{(U, Z)}$ is defined as follows:

For a fuzzy soft expert set $(G, B)$ in $\overline{\left(Y, Z^{\prime}\right)}, f^{-1}\left(G_{\delta}, B\right)$ is a fuzzy soft expert set in $\overline{(U, Z)}$ obtained as follows:

$$
f^{-1}\left(G_{\delta}, A\right)(\alpha)(u)=\left\{\begin{array}{lc}
G(s(\alpha))(r(u)) & \text { if } s(\alpha) \in B, \\
0 & \text { otherwise. }
\end{array}\right.
$$

For $\alpha \in s^{-1}(B) \subseteq Z$ and $u \in U . f(F, A)$ is called a fuzzy soft expert inverse image of the fuzzy soft expert set $(F, A)$.

Example 9 Let $U=\left\{u_{1}, u_{2}, u_{3}\right\}, Y=\left\{y_{1}, y_{2}, y_{3}\right\}$ and let

$$
A \subseteq Z=\left\{\left(e_{1}, p, 1\right),\left(e_{2}, p, 0\right),\left(e_{3}, p, 1\right),\left(e_{1}, q, 1\right),\left(e_{2}, q, 1\right),\left(e_{3}, q, 0\right),\left(e_{1}, r, 0\right),\left(e_{2}, r, 1\right),\left(e_{3}, r, 1\right)\right\}
$$


Table 3. $s_{j}=c_{j}-k_{j}$.

\begin{tabular}{ccc}
\hline$c_{j}=\sum_{i} u_{i j}$ & $k_{j}=\sum_{i} u_{i j}$ & $s_{j}=c_{j}-k_{j}$ \\
\hline$c_{1}=3.6$ & $k_{1}=3.9$ & $s_{1}=-0.3$ \\
$c_{2}=3.3$ & $k_{2}=4$ & $s_{2}=-0.7$ \\
$c_{3}=4$ & $k_{3}=4.1$ & $s_{3}=-0.1$ \\
$c_{4}=4.6$ & $k_{4}=3.2$ & $s_{4}=1.4$ \\
\hline
\end{tabular}

and

$$
A^{\prime} \subseteq Z^{\prime}=\left\{\left(e_{1}^{\prime}, p^{\prime}, 1\right),\left(e_{2}^{\prime}, p^{\prime}, 0\right),\left(e_{1}^{\prime}, q^{\prime}, 1\right),\left(e_{2}^{\prime}, q^{\prime}, 1\right),\left(e_{1}^{\prime}, r^{\prime}, 0\right),\left(e_{2}^{\prime}, r^{\prime}, 1\right)\right\}
$$

Suppose that $\overline{(U, A)}$ and $\overline{\left(Y, A^{\prime}\right)}$ are fuzzy soft expert classes. Define $r: U \rightarrow Y$ and $s: A \rightarrow A^{\prime}$ as follows:

$$
\begin{gathered}
r\left(u_{1}\right)=y_{1}, r\left(u_{2}\right)=y_{3}, r\left(u_{3}\right)=y_{2}, \\
s\left(e_{1}, p, 1\right)=\left(e_{2}^{\prime}, r^{\prime}, 1\right), s\left(e_{2}, p, 0\right)=\left(e_{1}^{\prime}, p^{\prime}, 1\right), s\left(e_{3}, p, 1\right)=\left(e_{1}^{\prime}, r^{\prime}, 0\right), \\
s\left(e_{1}, q, 1\right)=\left(e_{2}^{\prime}, q^{\prime}, 1\right), s\left(e_{2}, q, 1\right)=\left(e_{2}^{\prime}, p^{\prime}, 0\right), s\left(e_{3}, q, 0\right)=\left(e_{1}^{\prime}, q^{\prime}, 1\right), \\
s\left(e_{1}, r, 0\right)=\left(e_{2}^{\prime}, q^{\prime}, 1\right), s\left(e_{2}, r, 1\right)=\left(e_{1}^{\prime}, p^{\prime}, 1\right), s\left(e_{3}, r, 1\right)=\left(e_{2}^{\prime}, p^{\prime}, 0\right) .
\end{gathered}
$$

Let $(F, A)$ and $\left(G, A^{\prime}\right)$ be two fuzzy soft expert sets over $U$ and $Y$ respectively such that

$$
\begin{aligned}
&(F, A)=\left\{\left(\left(e_{1}, p, 1\right),\left\{\frac{u_{1}}{0.5}, \frac{u_{2}}{0.6}, \frac{u_{3}}{0.4}\right\}\right),\left(\left(e_{1}, q, 1\right),\left\{\frac{u_{1}}{0.1}, \frac{u_{2}}{0.4}, \frac{u_{3}}{0.2}\right\}\right),\right.\left(\left(e_{2}, q, 1\right),\left\{\frac{u_{1}}{0.3}, \frac{u_{2}}{0.5}, \frac{u_{3}}{0.7}\right\}\right),\left(\left(e_{2}, r, 1\right),\left\{\frac{u_{1}}{0.2}, \frac{u_{2}}{0.6}, \frac{u_{3}}{0.3}\right\}\right), \\
&\left(\left(e_{3}, p, 1\right),\left\{\frac{u_{1}}{0.1}, \frac{u_{2}}{0.4}, \frac{u_{3}}{0.6}\right\}\right),\left(\left(e_{3}, r, 1\right),\left\{\frac{u_{1}}{0.4}, \frac{u_{2}}{0.9}, \frac{u_{3}}{0.8}\right\}\right), \\
&\left(\left(e_{1}, r, 0\right),\left\{\frac{u_{1}}{0.2}, \frac{u_{2}}{0.2}, \frac{u_{3}}{0.6}\right\}\right),\left(\left(e_{2}, p, 0\right),\left\{\frac{u_{1}}{0.8}, \frac{u_{2}}{0.7}, \frac{u_{3}}{0.6}\right\}\right), \\
&\left.\left(\left(e_{3}, q, 0\right),\left\{\frac{u_{1}}{0.7}, \frac{u_{2}}{0.5}, \frac{u_{3}}{0.2}\right\}\right),\right\} \\
&\left(G, A^{\prime}\right)=\left\{\left(\left(e_{1}^{\prime}, p^{\prime}, 1\right),\left\{\frac{y_{1}}{0.7}, \frac{y_{2}}{0.3}, \frac{y_{3}}{0.8}\right\}\right),\left(\left(e_{2}^{\prime}, p^{\prime}, 0\right),\left\{\frac{y_{1}}{0.2}, \frac{y_{2}}{0.5}, \frac{y_{3}}{0.1}\right\}\right),\right. \\
&\left(\left(e_{1}^{\prime}, q^{\prime}, 1\right),\left\{\frac{y_{1}}{0.4}, \frac{y_{2}}{0.7}, \frac{y_{3}}{0.2}\right\}\right),\left(\left(e_{2}^{\prime}, q^{\prime}, 1\right),\left\{\frac{y_{1}}{0.8}, \frac{y_{2}}{0.2}, \frac{y_{3}}{0.5}\right\}\right), \\
&\left.\left(\left(e_{1}^{\prime}, r^{\prime}, 0\right),\left\{\frac{y_{1}}{0.3}, \frac{y_{2}}{0.1}, \frac{y_{3}}{0.2}\right\}\right),\left(\left(e_{2}^{\prime}, r^{\prime}, 1\right),\left\{\frac{y_{1}}{0.5}, \frac{y_{2}}{0.5}, \frac{y_{3}}{0.3}\right\}\right)\right\}
\end{aligned}
$$

Then we define a mapping $f: \overline{(U, Z)} \rightarrow \overline{\left(Y, Z^{\prime}\right)}$ as follows:

For a soft expert set $(F, A)$ in $(X, Z),(f(F, A), K)$ is a soft expert set in $\left(Y, Z^{\prime}\right)$ where $K=s(A)=\left\{\left(e_{1}^{\prime}, p^{\prime}, 1\right),\left(e_{2}^{\prime}, p^{\prime}, 0\right),\left(e_{1}^{\prime}, q^{\prime}, 1\right),\left(e_{2}^{\prime}, q^{\prime}, 1\right),\left(e_{1}^{\prime}, r^{\prime}, 0\right)\right\}$ and is obtained as follows:

$$
\begin{aligned}
& f(F, A)\left(e_{1}^{\prime}, p^{\prime}, 1\right)\left(y_{1}\right)=\bigvee_{x \in r^{-1}\left(y_{1}\right)}\left(\bigvee_{\alpha} F(\alpha)\right)=\bigvee_{x \in\left\{u_{1}\right\}}\left(\underset{\alpha \in\left\{\left(e_{2}, p, 0\right),\left(e_{2}, r, 1\right)\right\}}{\bigvee} F(\alpha)\right)=0.8 . \\
& f(F, A)\left(e_{1}^{\prime}, p^{\prime}, 1\right)\left(y_{2}\right)=\bigvee_{x \in r^{-1}\left(y_{2}\right)}\left(\bigvee_{\alpha} F(\alpha)\right)=\bigvee_{x \in\left\{u_{3}\right\}}\left(\bigvee_{\alpha \in\left\{\left(e_{2}, p, 0\right),\left(e_{2}, r, 1\right)\right\}} F(\alpha)\right)=0.6 .
\end{aligned}
$$




$$
f(F, A)\left(e_{1}^{\prime}, p^{\prime}, 1\right)\left(y_{3}\right)=\bigvee_{x \in r^{-1}\left(y_{3}\right)}\left(\bigvee_{\alpha} F(\alpha)\right)=\bigvee_{x \in\left\{u_{2}\right\}}\left(\underset{\alpha \in\left\{\left(e_{2}, p, 0\right),\left(e_{2}, r, 1\right)\right\}}{\bigvee} F(\alpha)\right)=0.7
$$

Then $f(F, A)\left(e_{1}^{\prime}, p^{\prime}, 1\right)=\left\{\frac{y_{1}}{0.8}, \frac{y_{2}}{0.6}, \frac{y_{3}}{0.7}\right\}$

$$
\begin{aligned}
& f(F, A)\left(e_{2}^{\prime}, p^{\prime}, 0\right)\left(y_{1}\right)=\bigvee_{x \in r^{-1}\left(y_{1}\right)}\left(\bigvee_{\alpha} F(\alpha)\right)=\bigvee_{x \in\left\{u_{1}\right\}}\left(\underset{\alpha \in\left\{\left(e_{2}, q, 1\right),\left(e_{3}, r, 1\right)\right\}}{\bigvee} F(\alpha)\right)=0.4 . \\
& f(F, A)\left(e_{2}^{\prime}, p^{\prime}, 0\right)\left(y_{2}\right)=\bigvee_{x \in r^{-1}\left(y_{2}\right)}\left(\bigvee_{\alpha} F(\alpha)\right)=\bigvee_{x \in\left\{u_{3}\right\}}\left(\underset{\alpha \in\left\{\left(e_{2}, p, 0\right),\left(e_{2}, r, 1\right)\right\}}{\bigvee} F(\alpha)\right)=0.8 . \\
& f(F, A)\left(e_{2}^{\prime}, p^{\prime}, 0\right)\left(y_{3}\right)=\bigvee_{x \in r^{-1}\left(y_{3}\right)}\left(\bigvee_{\alpha} F(\alpha)\right)=\bigvee_{x \in\left\{u_{2}\right\}}\left(\underset{\alpha \in\left\{\left(e_{2}, p, 0\right),\left(e_{2}, r, 1\right)\right\}}{\bigvee} F(\alpha)\right)=0.9 .
\end{aligned}
$$

Then $f(F, A)\left(e_{2}^{\prime}, p^{\prime}, 0\right)=\left\{\frac{y_{1}}{0.4}, \frac{y_{2}}{0.8}, \frac{y_{3}}{0.9}\right\}$

$$
\begin{aligned}
& f(F, A)\left(e_{1}^{\prime}, q^{\prime}, 1\right)\left(y_{1}\right)=\bigvee_{x \in r^{-1}\left(y_{1}\right)}\left(\bigvee_{\alpha} F(\alpha)\right)=\bigvee_{x \in\left\{u_{1}\right\}}\left(\underset{\alpha \in\left\{\left(e_{3}, q, 0\right)\right\}}{\bigvee} F(\alpha)\right)=0.7 . \\
& f(F, A)\left(e_{1}^{\prime}, q^{\prime}, 1\right)\left(y_{2}\right)=\bigvee_{x \in r^{-1}\left(y_{2}\right)}\left(\bigvee_{\alpha} F(\alpha)\right)=\bigvee_{x \in\left\{u_{3}\right\}}\left(\bigvee_{\alpha \in\left\{\left(e_{3}, q, 0\right)\right\}} F(\alpha)\right)=0.2 . \\
& f(F, A)\left(e_{1}^{\prime}, q^{\prime}, 1\right)\left(y_{3}\right)=\bigvee_{x \in r^{-1}\left(y_{3}\right)}\left(\bigvee_{\alpha} F(\alpha)\right)=\bigvee_{x \in\left\{u_{2}\right\}}\left(\bigvee_{\alpha \in\left\{\left(e_{3}, q, 0\right)\right\}}^{\bigvee} F(\alpha)\right)=0.5 .
\end{aligned}
$$

Then $f(F, A)\left(e_{1}^{\prime}, q^{\prime}, 1\right)=\left\{\frac{y_{1}}{0.7}, \frac{y_{2}}{0.2}, \frac{y_{3}}{0.5}\right\}$

$$
\begin{aligned}
& f(F, A)\left(e_{2}^{\prime}, q^{\prime}, 1\right)\left(y_{1}\right)=\bigvee_{x \in r^{-1}\left(y_{1}\right)}\left(\bigvee_{\alpha} F(\alpha)\right)=\bigvee_{x \in\left\{u_{1}\right\}}\left(\bigvee_{\alpha \in\left\{\left(e_{1}, q, 1\right),\left(e_{1}, r, 0\right)\right\}} F(\alpha)\right)=0.2 . \\
& f(F, A)\left(e_{2}^{\prime}, q^{\prime}, 1\right)\left(y_{2}\right)=\bigvee_{x \in r^{-1}\left(y_{2}\right)}\left(\bigvee_{\alpha} F(\alpha)\right)=\bigvee_{x \in\left\{u_{3}\right\}}\left(\bigvee_{\alpha \in\left\{\left(e_{1}, q, 1\right),\left(e_{1}, r, 0\right)\right\}} F(\alpha)\right)=0.6 . \\
& f(F, A)\left(e_{2}^{\prime}, q^{\prime}, 1\right)\left(y_{3}\right)=\bigvee_{x \in r^{-1}\left(y_{3}\right)}\left(\bigvee_{\alpha} F(\alpha)\right)=\bigvee_{x \in\left\{u_{2}\right\}}\left(\underset{\alpha \in\left\{\left(e_{1}, q, 1\right),\left(e_{1}, r, 0\right)\right\}}{\bigvee} F(\alpha)\right)=0.4 .
\end{aligned}
$$

Then $f(F, A)\left(e_{2}^{\prime}, q^{\prime}, 1\right)=\left\{\frac{y_{1}}{0.2}, \frac{y_{2}}{0.6}, \frac{y_{3}}{0.4}\right\}$

$$
\begin{aligned}
& f(F, A)\left(e_{1}^{\prime}, r^{\prime}, 0\right)\left(y_{1}\right)=\bigvee_{x \in r^{-1}\left(y_{1}\right)}\left(\bigvee_{\alpha} F(\alpha)\right)=\bigvee_{x \in\left\{u_{1}\right\}}\left(\bigvee_{\alpha \in\left\{e_{3}, p, 1\right\}} F(\alpha)\right)=0.1 \\
& f(F, A)\left(e_{2}^{\prime}, q^{\prime}, 1\right)\left(y_{2}\right)=\bigvee_{x \in r^{-1}\left(y_{2}\right)}\left(\bigvee_{\alpha} F(\alpha)\right)=\bigvee_{x \in\left\{u_{3}\right\}}\left(\bigvee_{\alpha \in\left\{e_{3}, p, 1\right\}} F(\alpha)\right)=0.6 . \\
& f(F, A)\left(e_{2}^{\prime}, q^{\prime}, 1\right)\left(y_{3}\right)=\bigvee_{x \in r^{-1}\left(y_{3}\right)}\left(\bigvee_{\alpha} F(\alpha)\right)=\bigvee_{x \in\left\{u_{2}\right\}}\left(\bigvee_{\alpha \in\left\{e_{3}, p, 1\right\}} F(\alpha)\right)=0.4
\end{aligned}
$$

Then $f(F, A)\left(e_{2}^{\prime}, q^{\prime}, 1\right)=\left\{\frac{y_{1}}{0.1}, \frac{y_{2}}{0.6}, \frac{y_{3}}{0.4}\right\}$

\section{Hence}




$$
\begin{aligned}
(f(F, A), K)= & \left(\left(e_{1}^{\prime}, p^{\prime}, 1\right),\left\{\frac{y_{1}}{0.8}, \frac{y_{2}}{0.6}, \frac{y_{3}}{0.7}\right\}\right),\left(\left(e_{2}^{\prime}, p^{\prime}, 0\right),\left\{\frac{y_{1}}{0.4}, \frac{y_{2}}{0.8}, \frac{y_{3}}{0.9}\right\}\right), \\
& \left(\left(e_{1}^{\prime}, q^{\prime}, 1\right),\left\{\frac{y_{1}}{0.7}, \frac{y_{2}}{0.2}, \frac{y_{3}}{0.5}\right\}\right),\left(\left(e_{2}^{\prime}, q^{\prime}, 1\right),\left\{\frac{y_{1}}{0.2}, \frac{y_{2}}{0.6}, \frac{y_{3}}{0.4}\right\}\right), \\
& \left.\left(\left(e_{1}^{\prime}, r^{\prime}, 0\right),\left\{\frac{y_{1}}{0.1}, \frac{y_{2}}{0.6}, \frac{y_{3}}{0.4}\right\}\right)\right\}
\end{aligned}
$$

Next for the soft expert inverse images, the mapping $f^{-1}:\left(Y, Z^{\prime}\right) \rightarrow(X, Z)$ is defined as follows: For a soft expert set $\left(G, A^{\prime}\right)$ in $\left(Y, Z^{\prime}\right),\left(f^{-1}\left(G, A^{\prime}\right), D\right)$ is a soft expert set in $(Y, Z)$ where

$$
D=s^{-1}\left(A^{\prime}\right)=\left\{\left(e_{2}, p, 0\right),\left(e_{2}, r, 1\right),\left(e_{2}, q, 1\right),\left(e_{3}, r, 1\right),\left(e_{3}, q, 0\right)\left(e_{1}, p, 1\right),\left(e_{1}, r, 0\right),\left(e_{3}, p, 1\right),\left(e_{1}, p, 1\right)\right\}
$$

and is obtained as follows:

$$
\begin{aligned}
& f^{-1}(G, B)\left(\left(e_{2}, p, 0\right)\right)\left(u_{1}\right)=G\left(s\left(e_{2}, p, 0\right)\right)\left(r\left(u_{1}\right)\right)=G\left(\left(e_{1}^{\prime}, p^{\prime}, 1\right)\right)\left(y_{1}\right)=0.7 . \\
& f^{-1}(G, B)\left(\left(e_{2}, p, 0\right)\right)\left(u_{2}\right)=G\left(s\left(e_{2}, p, 0\right)\right)\left(r\left(u_{2}\right)\right)=G\left(\left(e_{1}^{\prime}, p^{\prime}, 1\right)\right)\left(y_{3}\right)=0.8 . \\
& f^{-1}(G, B)\left(\left(e_{2}, p, 0\right)\right)\left(u_{3}\right)=G\left(s\left(e_{2}, p, 0\right)\right)\left(r\left(u_{3}\right)\right)=G\left(\left(e_{1}^{\prime}, p^{\prime}, 1\right)\right)\left(y_{2}\right)=0.3 .
\end{aligned}
$$

Then $f^{-1}(G, B)\left(\left(e_{2}, p, 0\right)\right)=\left\{\frac{u_{1}}{0.7}, \frac{u_{2}}{0.8}, \frac{u_{3}}{0.3}\right\}$

$$
\begin{aligned}
& f^{-1}(G, B)\left(\left(e_{2}, r, 1\right)\right)\left(u_{1}\right)=G\left(s\left(e_{2}, r, 1\right)\right)\left(r\left(u_{1}\right)\right)=G\left(\left(e_{1}^{\prime}, p^{\prime}, 1\right)\right)\left(y_{1}\right)=0.7 . \\
& f^{-1}(G, B)\left(\left(e_{2}, r, 1\right)\right)\left(u_{2}\right)=G\left(s\left(e_{2}, r, 1\right)\right)\left(r\left(u_{2}\right)\right)=G\left(\left(e_{1}^{\prime}, p^{\prime}, 1\right)\right)\left(y_{3}\right)=0.8 . \\
& f^{-1}(G, B)\left(\left(e_{2}, r, 1\right)\right)\left(u_{3}\right)=G\left(s\left(e_{2}, r, 1\right)\right)\left(r\left(u_{3}\right)\right)=G\left(\left(e_{1}^{\prime}, p^{\prime}, 1\right)\right)\left(y_{2}\right)=0.3 .
\end{aligned}
$$

Then $f^{-1}(G, B)\left(\left(e_{2}, r, 1\right)\right)=\left\{\frac{u_{1}}{0.7}, \frac{u_{2}}{0.8}, \frac{u_{3}}{0.3}\right\}$

By similar calculations, consequently, we get

$$
\begin{aligned}
\left(f^{-1}\left(G, A^{\prime}\right), D\right)=\{ & \left(\left(e_{2}, p, 0\right),\left\{\frac{u_{1}}{0.7}, \frac{u_{2}}{0.8}, \frac{u_{3}}{0.3}\right\}\right),\left(\left(e_{2}, r, 1\right),\left\{\frac{u_{1}}{0.7}, \frac{u_{2}}{0.8}, \frac{u_{3}}{0.3}\right\}\right), \\
& \left(\left(e_{2}, q, 1\right),\left\{\frac{u_{1}}{0.2}, \frac{u_{2}}{0.1}, \frac{u_{3}}{0.5}\right\}\right),\left(\left(e_{3}, r, 1\right),\left\{\frac{u_{1}}{0.2}, \frac{u_{2}}{0.1}, \frac{u_{3}}{0.5}\right\}\right), \\
& \left(\left(e_{3}, q, 0\right),\left\{\frac{u_{1}}{0.4}, \frac{u_{2}}{0.2}, \frac{u_{3}}{0.7}\right\}\right),\left(\left(e_{1}, q, 1\right),\left\{\frac{u_{1}}{0.8}, \frac{u_{2}}{0.5}, \frac{u_{3}}{0.2}\right\}\right), \\
& \left(\left(e_{1}, r, 0\right),\left\{\frac{u_{1}}{0.8}, \frac{u_{2}}{0.5}, \frac{u_{3}}{0.2}\right\}\right),\left(\left(e_{3}, p, 1\right),\left\{\frac{u_{1}}{0.8}, \frac{u_{2}}{0.5}, \frac{u_{3}}{0.2}\right\}\right), \\
& \left.\left(\left(e_{1}, p, 1\right),\left\{\frac{u_{1}}{0.3}, \frac{u_{2}}{0.2}, \frac{u_{3}}{0.1}\right\}\right)\right\} .
\end{aligned}
$$

Definition 27 Let $f: \overline{(U, X)} \rightarrow \overline{\left(Y, X^{\prime}\right)}$ be a mapping and $(F, A)$ and $(G, B)$ fuzzy soft expert sets in $\overline{(U, X)}$. Then for $\beta \in X^{\prime}, \quad y \in Y$, the fuzzy soft expert union and intersection of fuzzy soft expert images $(F, A)$ and $(G, B)$ are defined as follows: 


$$
\begin{aligned}
& \left(f(F, A) \tilde{\vee}_{f}(G, B)\right)(\beta)(y)=f(F, A)(\beta)(y) \tilde{\vee}_{f(G, B)(\beta)(y) .} \\
& \left(f(F, A) \tilde{\wedge}_{f}(G, B)\right)(\beta)(y)=f(F, A)(\beta)(y) \tilde{\wedge}_{f}(G, B)(\beta)(y) .
\end{aligned}
$$

Definition 28 Let $f: \overline{(U, X)} \rightarrow \overline{\left(Y, X^{\prime}\right)}$ be a mapping and $(F, A)$ and $(G, B)$ fuzzy soft expert sets in $\left(Y, X^{\prime}\right)$. Then for $\alpha \in X, u \in U$, the fuzzy soft expert union and intersection of fuzzy soft expert inverse images $(F, A)$ and $(G, B)$ are defined as follows:

$$
\begin{aligned}
& \left(f^{-1}(F, A) \tilde{\vee}_{f}^{-1}(G, B)\right)(\alpha)(u)=f^{-1}(F, A)(\alpha)(u) \tilde{\nabla} f^{-1}(G, B)(\alpha)(u) . \\
& \left(f^{-1}(F, A) \tilde{\wedge} f^{-1}(G, B)\right)(\alpha)(u)=f^{-1}(F, A)(\alpha)(u) \tilde{\wedge} f^{-1}(G, B)(\alpha)(u) .
\end{aligned}
$$

Theorem 1 Let $f: \overline{(U, X)} \rightarrow \overline{\left(Y, X^{\prime}\right)}$ be a mapping. Then for fuzzy soft expert sets $(F, A)$ and $(G, B)$ in the fuzzy soft expert class $\overline{(U, X)}$, [a.]

1. $f(\varnothing)=\varnothing$.

2. $f(X) \subseteq Y$.

3. $f((F, A) \tilde{\nabla}(G, B))=f(F, A) \widetilde{\nabla} f(G, B)$.

4. $f((F, A) \tilde{\wedge}(G, B)) \subseteq f(F, A) \tilde{\wedge}_{f}(G, B)$.

5. If $(F, A) \subseteq(G, B)$, then $f(F, A) \subseteq f(G, B)$,

Proof For (a), (b) and (e) the proof is trivial, so we just give the proof of (c) and (d).

c. For $\beta \in X^{\prime}$ and $y \in Y$, we want to prove that

$$
f((F, A) \tilde{\vee}(G, B))(\beta)(y)=f(F, A)(\beta)(y) \tilde{\vee}_{f}(G, B)(\beta)(y)
$$

For left hand side, consider $f((F, A) \widetilde{\vee}(G, B))(\beta)(y)=f(H, A \cup B)(\beta)(y)$. Then

$$
f(H, A \cup B)(\beta)(y)=\left\{\begin{array}{lc}
\bigvee_{x \in r^{-1}(y)}\left(\bigvee_{\alpha} H(\alpha)\right), & \text { if } r^{-1}(y) \text { and } s^{-1}(\beta) \cap(A \cup B) \neq \varnothing, \\
(0,0) & \text { otherwise. }
\end{array}\right.
$$

such that $H(\alpha)=F(\alpha) \tilde{U} G(\alpha)$ where $\tilde{U}$ an interval-valued fuzzy union.

Considering only the non-trivial case, then Equation 0.1 becomes:

$$
f(H, A \cup B)(\beta)(y)=\bigvee_{x \in r^{-1}(y)}(\bigvee(F(\alpha) \tilde{U} G(\alpha))) .
$$

For right hand side and by using Definition 27, we have

$$
\begin{aligned}
& (f(F, A) \tilde{\bigvee} f(G, B))(\beta)(y) \\
& =f(F, A)(\beta)(y) \bigvee f(G, B)(\beta)(y) \\
& =\left(\bigvee_{x \in r^{-1}(y)}\left(\bigvee_{\alpha \in s^{-1}(\beta) \cap A} F(\alpha)\right)(x)\right) \bigvee\left(\bigvee_{x \in r^{-1}(y)}\left(\bigvee_{\alpha \in s^{-1}(\beta) \cap B} G(\alpha)\right)\right) \\
& =\bigvee_{x \in r^{-1}(y)} \bigvee_{\alpha \in s^{-1}(\beta) \cap(A \cup B)}(F(\alpha) \bigvee G(\alpha))=\bigvee_{x \in r^{-1}(y)}(\bigvee(F(\alpha) \tilde{U} G(\alpha)))
\end{aligned}
$$


From Equations (1.1) and (1.3), we get (c).

d. For $\beta \in X^{\prime}$ and $y \in Y$, and using Definition 27, we have

$$
\begin{aligned}
& f((F, A) \tilde{\wedge}(G, B))(\beta)(y) \\
= & f(H, A \cup B)(\beta)(y) \\
= & \bigvee_{x \in r^{-1}(y)}\left(\bigvee_{\alpha \in s^{-1}(\beta) \cap(A \cup B)} H(\alpha)\right)(x) \\
= & \bigvee_{x \in r^{-1}(y)}\left(\bigvee_{\alpha \in s^{-1}(\beta) \cap(A \cup B)} F(\alpha) \tilde{\cap} G(\alpha)\right)(x) \\
= & \bigvee_{x \in r^{-1}(y)}\left(\underset{\alpha \in s^{-1}(\beta) \cap(A \cup B)}{\bigvee} F(\alpha)(x) \tilde{\cap} G(\alpha)(x)\right) \\
\subseteq & \left.\bigvee_{x \in r^{-1}(y)}\left(\bigvee_{\alpha \in s^{-1}(\beta) \cap A} F(\alpha)(x)\right)\right) \wedge\left(\bigvee_{x \in r^{-1}(y)}\left(\bigvee_{\alpha \in s^{-1}(\beta) \cap B} G(\alpha)(x)\right)\right) \\
= & f((F, A))(\beta)(y) \wedge((G, B))(\beta)(y) \\
= & (f(F, A) \wedge f(G, B))(\beta)(y) .
\end{aligned}
$$

This gives (d).

Theorem 2 Let $f: \overline{(U, X)} \rightarrow \overline{\left(Y, X^{\prime}\right)}$ be mapping. Then for fuzzy soft experts $(F, A),(G, B)$ in the fuzzy soft expert class $\left(X, E^{\prime}\right)$, we have:

1. $f^{-1}\left(\varnothing_{0}\right)=\varnothing_{0}$.

2. $f^{-1}\left(A_{1}\right)=B_{1}$.

3. $f^{-1}((F, A) \tilde{\vee}(G, B))=f^{-1}(F, A) \tilde{\nabla} f^{-1}(G, B)$.

4. $f^{-1}((F, A) \tilde{\wedge}(G, B))=f^{-1}(F, A) \tilde{\wedge} f^{-1}(G, B)$.

5. If $(F, A) \subseteq(G, B)$, then $f^{-1}(F, A) \subseteq f^{-1}(G, B)$.

Proof We use the same method as in the previous proof.

\section{Conclusion}

In this paper, we have introduced the concept of fuzzy soft expert set which is more effective and useful and studied some of its properties. Also the basic operations on fuzzy soft expert set namely complement, union, intersection, AND and OR have been defined. An application of this theory has been given to solve a decisionmaking problem. We also studied a mapping on fuzzy soft expert classes and its properties.

\section{Acknowledgements}

The authors would like to acknowledge the financial support received from Universiti Kebangsaan Malaysia under the research grant AP-2013-009.

\section{References}

[1] Molodtsov, D. (1999) Soft Set Theory-First Results. Computers \& Mathematics with Applications, 37, $19-31$. http://dx.doi.org/10.1016/S0898-1221(99)00056-5

[2] Chen, D., Tsang, E.C.C., Yeung, D.S. and Wang, X. (2005) The Parameterization Reduction of Soft Sets and Its Application. Computers \& Mathematics with Applications, 49, 757-763. http://dx.doi.org/10.1016/j.camwa.2004.10.036

[3] Maji, P.K., Roy, A.R. and Biswas, R. (2003) Soft Set Theory. Computers \& Mathematics with Applications, 54, 555562. http://dx.doi.org/10.1016/S0898-1221(03)00016-6 
[4] Maji, P.K., Roy, A.R. and Biswas, R. (2002) An Application of Soft Sets in a Decision Making Problem. Computers \& Mathematics with Applications, 44, 1077-1083. http://dx.doi.org/10.1016/S0898-1221(02)00216-X

[5] Maji, P.K., Roy, A.R. and Biswas, R. (2001) Fuzzy Soft Sets. Journal of Fuzzy Mathematics, 9, 589-602.

[6] Roy, R. and Maji, P.K. (2007) A Fuzzy Soft Set Theoretic Approach to Decision Making Problems. Journal of Computational and Applied Mathematics, 203, 412-418. http://dx.doi.org/10.1016/j.cam.2006.04.008

[7] Alkhazaleh, S., Salleh, A.R. and Hassan, N. (2011) Soft Multisets Theory. Applied Mathematical Sciences, 5, 35613573.

[8] Alkhazaleh, S., Salleh, A.R. and Hassan, N. (2011) Fuzzy Parameterized Interval-Valued Fuzzy Soft Set. Applied Mathematical Sciences, 5, 3335-3346.

[9] Alkhazaleh, S., Salleh, A.R. and Hassan, N. (2011) Possibility Fuzzy Soft Set. Advances in Decision Sciences, 2011, $18 \mathrm{p}$.

[10] Alkhazaleh, S. and Salleh, A.R. (2011) Soft Expert Sets. Advances in Decision Sciences, 2011, 15 p. 